\title{
THE PHYSIOLOGICAL EFFECTS OF INTRAVENOUS ANAESTHESIA ON MAN
}

\author{
Aluen B Dobrin, M D., and Gordon M. WyanT, F.F.A.R.C.s."
}

\section{INTRODUCTION}

KEATING noted in 1956 that intravenous anaesthetics cause as many sudden unexplained deaths as did chloroform (1). From recent extensive reviews it appears that estımated death rates from intravenous anaesthetics have been conservative $(2,3,4)$. The introduction of new or "better" intravenous agents must therefore be most carefully assessed in the most reliable and valid parameters available for study.

Theory and data recently procured have provided the clnical anaesthetist with some fundamental facts which should form a basis for comparing new agents and for the improved menagement of intravenous inductons of anaesthesia.

First, and foremost, the effect of premedicant drugs administered to the patent before the intravenous anaesthétic agent must be determined. For this reason, all drugs tested should be given preferably to unpremedicated subjects in order to separate the changes in vasomotor and haemodynamic stability as well as in respiration which occur with premedicant drugs. Clinicians have almost totally ignored or have not included these effects in their studies of changes (5-11).

In addition to theoretical considerations of the intrinsic activity of each drug, the affinity of drugs for various tissues and the effect of drug interaction, it is essential that the anaesthetist also note the physiological age, the state of nutrition and hydration as well as the state of electrolytes in the subjects whom he studies.

To compare the effects of drugs under clinical tests in a group of patients in whom. the above factors vary widely is completely invalid. To procure the usual average series of unselected cases may merely add to the variability of the basic conditions and throw in the less common disturbing factors of varying sensitivity.

Other factors of vital concern, particularly with respect to the ultra-short acting intravenous agents, are the phenomena of cumulation, of acute tolerance,

"From the Department of Anaesthesia, University of Saskatchewan College of Medicine and University Hospital, Saskatoon, Canada

Supplies of drugs were kindly made avallable by the following firms sodium thopental (Pentothal@) by Abbott Laboratones, Montreal, PQ, thiamylal sodıum (Surital $($ ) by Parke, Davis and Company Ltd, Walkerville, Ontarı, hexobarbital sodum (Evipal(B) by Winthrop Stearns Inc, New York, methitural sodium (Neraval@) by Schering Corporation, Montreal, P Q, buthalitone sodium (Transithal@) by Poulenc Ltd, Montreal, P Q, Comzound 25398 by Eli Lilly and Company, Indianapols, Ind, and MRD-125 (Dolitrone®) sy the Wm S Merrill Company, Cincinnat, Oho, US A.

The Wm S Merrill Company, Poulenc Ltd, Schenng Corporation and Abbott Laboratories assisted in this study by a grant-in-ard 
and perhaps also of tachyphylaxis Since the intravenous anaesthetics reach a very rapid peak of concentration sequentially in the blood, the brain, and the heart, at a rate which is almost synchronous with the curculation tome, and then are rapidly redistributed to the rest of the body, the effect of this inital distribution must be evaluated when severdl drugs are to be compared. This evaluation is very imporant, because the cerebral blood flow is maintamed even when a state of shock is impending or present Therefore, the effect of intravenous dugs on the brain will vary in an inverse relationship to the cardiac output. Subsequent injections of a drug under test produce a greater effect on the central nervous system and a lesser effect on the haemodynamics and cannot provide a valid comparıson. The cumulative effects or tachyphylaxis further confuse this picture.

As for the phenomenon of acute tolerance, Dundee and his associates have shown that the awakening tme from intravenous anaesthesia (thiopental) is primarily determined by the initial or acute dose of the intravenous anaesthetic (12).

The physiological effect of intravenous anaesthetic agents on haemodynamics must be considered of primary importance to the clmical anaesthetist, for a large proportion of deaths attributable to inductions of intravenous anaesthetic are caused by both respiratory and cardiovascular depression. The respiratory depression is quite easily supported, while the cardiovascular depression frequently cannot be reversed by vasopressors, by atropine and other supportive measures, especially in elderly or debilitated patients. As many' new agents are becoming avallable, it is essential to evaluate these carefully on healthy subjects prior to employing them in clinical trials. Since the greatest difficulties arise immediately after induction of anaesthesia, the most pertınent information should be obtained at this time, rather than after a so-called steady state develops. However these data are procured, the investigator must either account for the gross alterations from the basal state in the patient prepared for an operation or a test study, or clearly evaluate the effect of apprehension or of the narcotic premedication on the parameters being investigated

One of the reasons for the paucity of data on the haemodynamic effects of anaesthetic agents is the technical difficulty encountered in procurmg such information in man Since a simple method was devised recently, it was deemed important to compare the effects of several intravenous anaesthetics on haemodynamics as well as on respiration of the same individual subjects. This comparison provided an integral part of the experimental and clinical evaluations of the newer anaesthetic agents which have been studied $(13,14)$

\section{Method}

For each subject, vital statistics (sex, age, body buld, height, weight and body surface area) were measured and noted Blood volume fractions, venous haematocrit and resting vital signs were recorded. Respiratory and haemodynamic parameters were estımated and calculated from metabolism tables 
IN'TRAVENOUIS ANAESTHETICS

\begin{tabular}{|c|c|}
\hline $\begin{array}{l}\text { TRANSITHAL } \\
\text { Na Buthalitone } \\
\text { (Poulenc) }\end{array}$ & Na-5-Allyl-5-isobutyl-thiobarbiturate \\
\hline $\begin{array}{l}\text { NERAVAL } \\
\text { No Mothitural } \\
\text { (Schering) }\end{array}$ & Na-5-(l-Methylbutyl)-5-2(methylthio) ethyl-2 thiobabiturate \\
\hline
\end{tabular}

Compound 25398

(Eli Lilly)

No I-Methyl-5-ally|-5-(1-methyl-2-pentynyl)barbifurate

DOLITRONE

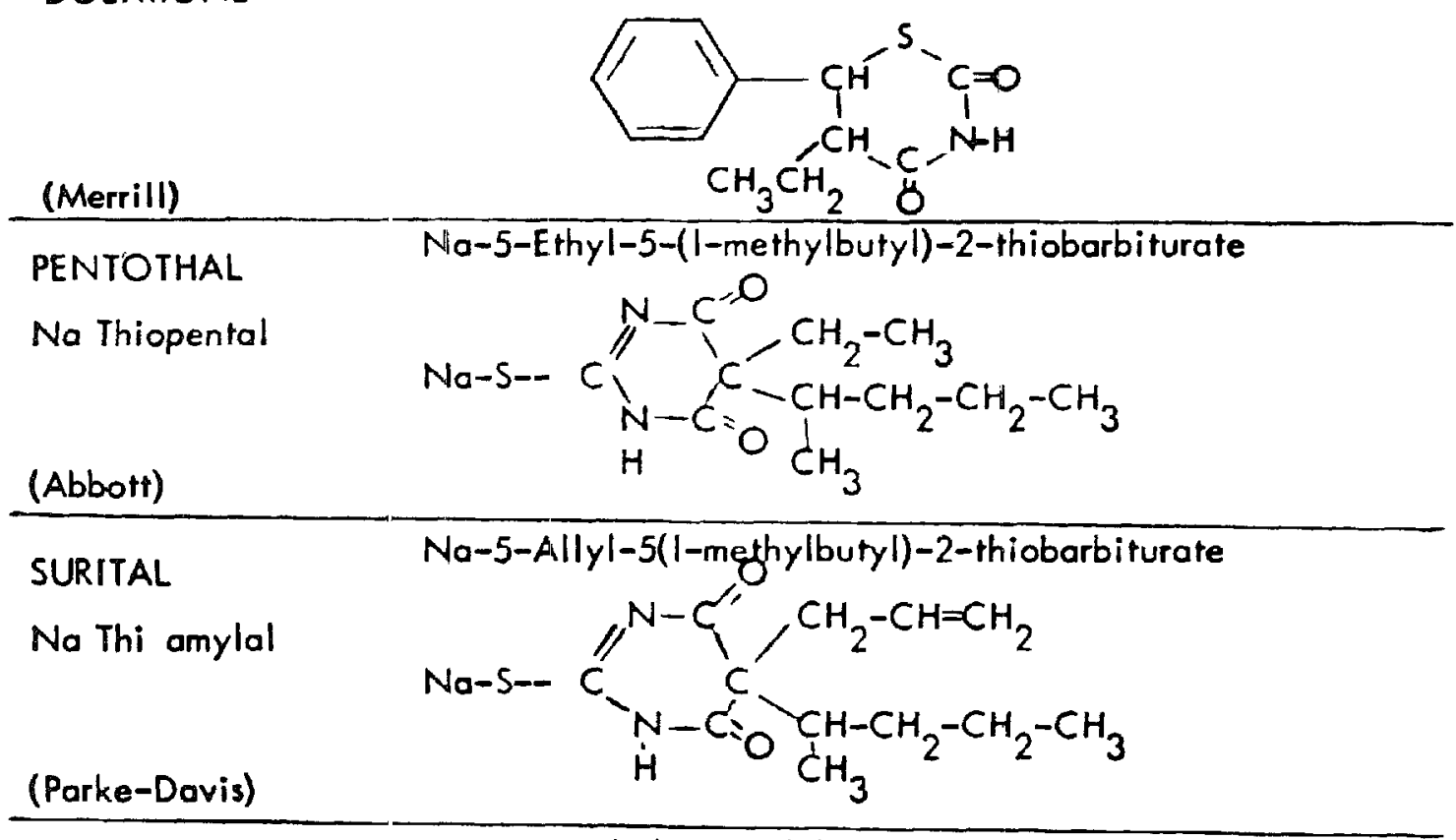

EVIPAL

Na Hexobarbital

Winthrop-Stearns)

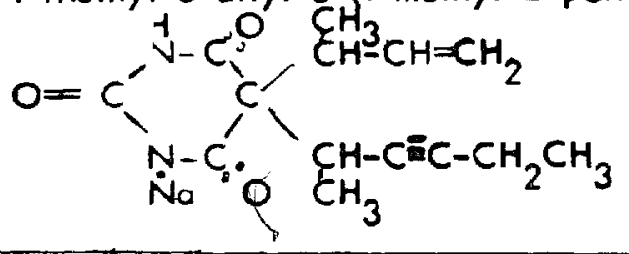

5-Ethyl-6-phenyl-M-thlazine-2,4-dione 
and from the nomograms of Radford and Jackson $(15,16)$. These data provided the base line for all changes recorded in the control period and in the acute and delayed test period. Alterations in recorded respiratory and haemodynamic parameters induced by apprehension or anxiety, and by the drugs under study, were then compared.

Data were obtained at weekly intervals from young, healthy, male subjects in a fasting state before and after intravenous administration of thiopental, thiamylal, hexobarbital, buthalitone, methitural, Compound 25398 and Dolitrone (Fig. 1). While the subject was requested to gaze at the ceiling, each freshly prepared drug was administered from a $20 \mathrm{ml}$. syringe in a 2.5 per cent solution through a $1 \frac{1 / 2}{2}$ "20-gauge needle at the rate of approximately $15 \mathrm{mg}$. per second until the eyelids closed involuntarily. Thereupon, an equal dose was injected as rapidly as pressure on the syringe would permit. Arrangement of recording data is shown in Figure 2.

\section{CALIBRATED PULMOTEST}

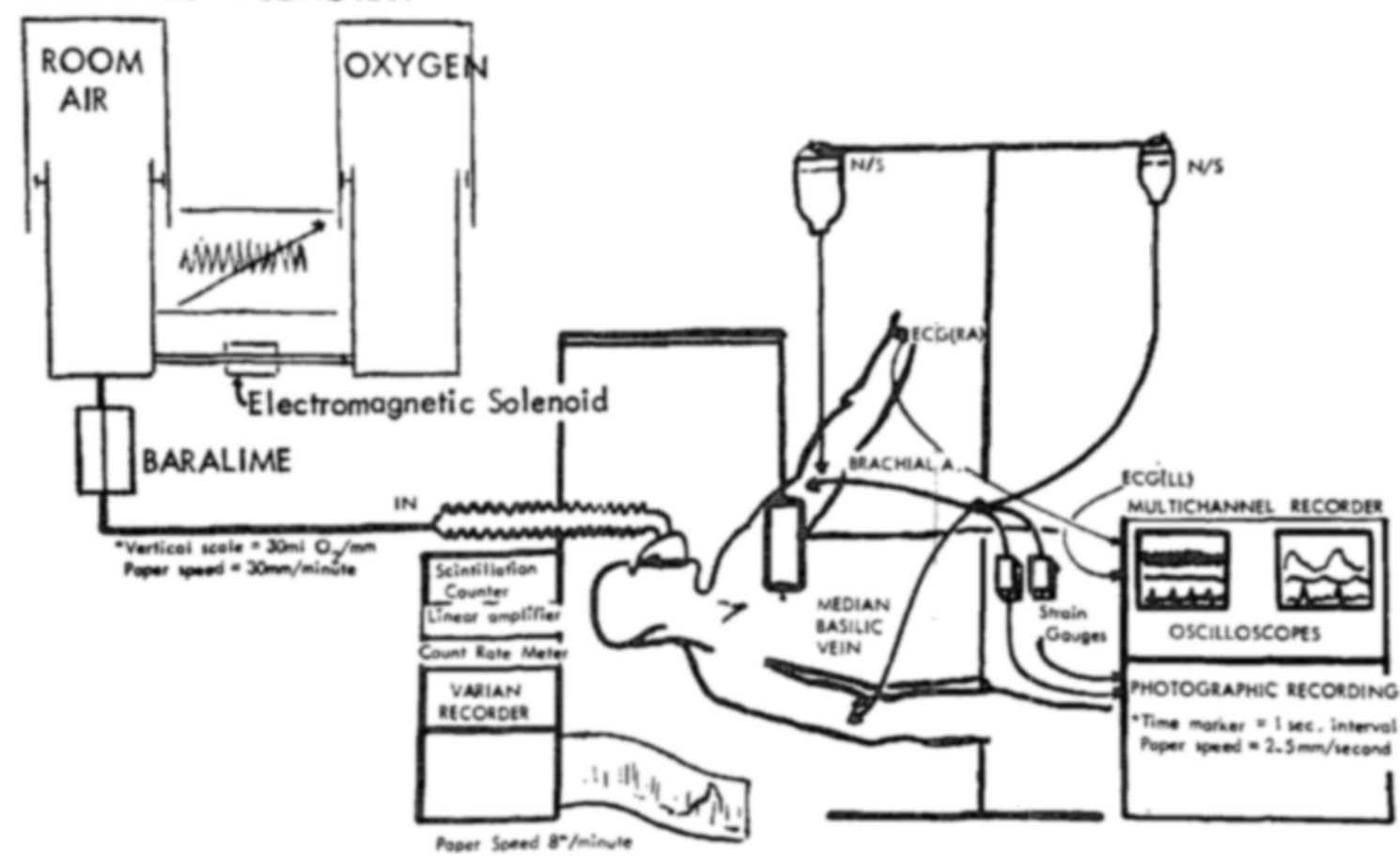

Figure 2. Arrangement of respiratory and haemodynamic recording equipment for study of intravenous anaesthetics.

As premedication was not given, it was realized that apprehension was the important factor in altering control values as compared to those of the reșting state $(17,18)$. This could not be avoided even in trained human subjects. No attempt was made to record electroencephalographic levels, as these were virtually "acute" experiments, and in previous studies a grossly erroneous indication of anaesthetic depth and reflex responsiveness during intravenous anaesthesia was observed $(13,19)$. It was further evident that electroencephalographic levels are not comparable among anaesthetics, even when their route of adminis- 
tration or mode of action may be similar. With any anaesthetic drug, if the "depth of anaesthesia" as determined by the electroencephalogram must be known within a reasonably steady blood level, collateral data must be obtained from repeated blood samples or a continuous monitor showing the arterial blood sugar, $\mathrm{pO}_{2}, \mathrm{pCO}_{2}$ and cerebral blood flow. Without this data, very wide variation in the blood level of the anaesthetic can exist, without significant alteration in the cortıcal potentials. Others are now beginning to report similar difficulties (20).

Determinations of blood volume were made on each subject prior to the drug tests, by means of a simple, accurate method which has been developed (21-25). Using human serum albumin iodinated with iodnne ${ }^{131}$ and radioactive sodium chromate containing chromium ${ }^{51}$, plasma volume and red cell volume were determined simultaneously $(26,27)$. In the first compound, the iodine is firmly bound to the protem and when injected intravenously, there is no transfer of activity to the plasma protems or to the red cells. Since the albumin is physically and chemically almost identical to the ordinary serum albumin, there is only a slow leakage from the capllary bed. Evans Blue (T1824) diffuses relatively rapidly from the curculating plasma, and repeated determinations within short time intervals are not possiole In the Evans Blue method, lipaemia or haemolysis will cause interference, but since only radioactivity is being measured in the 1odine ${ }^{131}$ serum albumin method, colour or turbidity is not a problem When a highly sensitive well-type scintillation counter is used, the dose of radioactivity given to the patient is negligible. With sodium chromate $\left(\mathrm{Na}_{2} \mathrm{CrO}_{4}\right)$ incubation with blood allows adsorption of the radioactive chromum ${ }^{51}$ by the cells and binding to the globulin in the cells The calculation of plasma and red cell volume is based on the dilution principle, just as in the Evans Blue method The general principles of this procedure are as follows:

1. (a) Venous blood ( $20 \mathrm{ml}$ ) from the subject is added to 150 microcuries of chromum ${ }^{51}$ and allowed to incubate at room temperature The subject's labelled cells are later remjected.

(b) Iodine ${ }^{131}$ labelled albumn is diluted, using sterle containers and sterle saline to an activity level of about 5 microcuries per $\mathrm{ml}$ Then, 5 to 10 microcuries of this dilution are injected intravenously into the subject, the syringe being well flushed with blood before being removed from the vein.

2 Standard dilute solutions are prepared by adding amounts exactly equal to that injected of the iodine ${ }^{131}$ and chromium ${ }^{51}$ to $4000 \mathrm{ml}$. and to $500 \mathrm{ml}$. volumetric flasks respectively and then bringing the solutions to the mark with distilled water.

3. Fifteen minutes after injection of $10 \mathrm{~d} n \mathrm{e}^{131}$ and chromium ${ }^{51}$ blood samples are withdrawn from the other arm and added to tubes contanning heparin or a dried potassium-ammonium-oxalate mixture.

4. Haematocrit determinations are made on the blood and on an aliquot centrifuged to obtain about $3 \mathrm{ml}$. of plasma

5. Then, 2 ml. samples of the standard solutions (2), the subject's blood ( 3 ) and plasma (4) are counted in a well-type scintillation counter.

6. After subtraction of background counts, a predicted blood volume may be estumated thus: 


$$
\mathrm{BV}=\frac{\text { Plasma volume }}{1-(\text { Hct } \times 09)}
$$

and then may be calculated thus:

Red cell volume $=\frac{\text { Counts per min of injected solution } / \mathrm{mll}}{\text { Net counts } / \mathrm{min} . / \mathrm{ml}} \times$ Volume of tagged cells injected Plasma volume $=\frac{\text { Counts } / \mathrm{mm} / \mathrm{ml} \text { of standard }}{\text { Counts } / \mathrm{mm} / \mathrm{ml} \text { plasma }} \times$ Volume of standard

Venous haematocrit was determined by centrifuging heparinized blood in Wintrobe tubes.

Normal values by this method $\mathrm{m} \mathrm{mg} / \mathrm{kg}$. in 25 other subjects were as follows: red cell volume, 22 to 37 (mean $28.5 \pm 4$ ), plasma volume, 33 to 60 (mean $430 \pm 68$ ). Total blood volume ranged from 55 to 90 The ratio of body haematocrit to venous haematocrit in 108 estumations was $089 \pm .05$ (27).

Arterial and venous blood pressures were measured directly from the brachial artery and the median basulic vein through $5 \mathrm{~cm}$. 18-gauge needles. These needles were connected to Statham strain gauges (Model P 23A) placed at the level of the right auricle, arbitrarily set at $100 \mathrm{~mm}$. above the level of the back as recommended by Lyons and associates (28) These pressures together with lead 2 of the electrocardıgram were recorded contunuously on two osclloscopes with an infinte frequency response One of these osculloscopes was set to amplify the complexes. The pressure heads had a frequency response of $185 \mathrm{c} \mathrm{p} \mathrm{s}$. through a $5 \mathrm{~cm}$ 20-gauge needle. Fry and assocrates have shown that the technical recording system employed is highly accurate and a stable system for recording pressure data (29). All tracings were photographed for permanent records and subsequent study

The mean pulse rate, mean venous pressure, mean systolic and drastolic blood pressure and mean pulse pressures were computed from the average of all pulse rates and arterial and venous blood pressures measured on the tracings for each subject. These measurements were made in a representative threeminute period when other data were recorded (cardiac output, oxygen consumption, respiration rate and tudal volume) before and immediately after the injection of the test drugs and again for five to thirty munutes during the awakening period when blood pressure, pulse rate and respiration had stabilized somewhat. From the tracings the mean arterial blood pressure was computed thus: mean arterial blood pressure $=$ mean diastolic $\mathrm{BP}+1 / 3$ (mean systolıc B P. - mean diastolic B P). It was found that this calculation provides essentially the same values as that determined by planimetry of the pulse waves.

Cardiac output Cardiac output was measured by the recording of radioactivity transients at the precordium by the external body counting of Iodine $^{131}$ as described by Veall and associates in 1954 and by Huff and associates in 1955 $(30,31)$. The technique described by the latter authors was followed exactly. A collimator with a diameter of $3 / 4$ inch was used on a thyroid counter. The detector was placed over the skin between the first and second ribs at the left 
parasternal line. The scintillation counter was connected to a count-rate meter and a Varian strip-chart recorder. For each determination 75 microcuries of Rihsa ${ }^{\circledR}$ in a small volume $(1 \mathrm{ml}$.) were injected directly into the same median basiluc vein in a single continuous motion at a rate not exceeding one second for the entire injection. The needle and vein were then flushed with normal saline. The uptake of Rihsa ${ }^{\circledR}$ was recorded until an equilibrium counting rate was observed on the calibrated recordıng chart. The initial fal in intensity of radioactivity following the first rise was extrapolated exponentially to the base line. The area under this curve was measured accurately by planimetry in square inches using a "Paragon" Compensating Polar Planimeter. The cardiac output in blood volumes per minute was then equal to:

$$
\frac{\text { Herght (in ) of equilibrium value } \times \text { record speed (1n } / \mathrm{mm} \text { ) }}{\text { Area ( } \mathrm{m}^{2} \text { of extrapolated curve) }} \text {. }
$$

The cardiac output in litres per minute was then determined from this estimation multiplied by the total blood volume which was determined initially. Theoretical considerations of this method will be amplified in the discussion. As estimations were made at weekly intervals, potassium iodide was prescribed to be taken by the subjects for a few days before and after each test, in order to prevent uptake of the radioactive iodine by the thyroid.

From the recorded determinations the following estimations were made:

1. Mean circulation time (MCT) was measured objectively by a method which would include the transit time through the heart and lungs $(32,33)$. This parameter is determined on the curve for cardiac output by measuring the first moment (or the centre of gravity) of the extrapolated curve. By definition

$$
M C T=\frac{\int_{T_{1}}^{\infty} c t d t}{\int_{T_{1}}^{\infty} c d t}
$$

(Cyvin's formula) (34). In practice this is done by summing the products $C_{1} T_{1}, C_{2} T_{2}, C_{3} T_{3}, C_{n} T_{n}$ and dividing this value by the sum of $C_{1}, C_{2}, C_{3}$ and $C_{n}$ as described by Hamilton in 1932 (35). This method is cumbersome and does not significantly increase the accuracy of that time measurement from the moment of starting injection of Rihsa into the median basilic vein $(x)$ until the peak activity is recorded over the precordium $(y)$, that is, on Figure 3 the $x y$ time interval is very nearly equal to the time interval from " $a$ " to the projection of the centre of gravity of the exponentially extrapolated curve on the abscissa. $X Y$ is very nearly equal to "ac" because the time concentration curve is skewed to the right (to the left on the recording). In six tracings checked at random, the mean difference between these two methods was less than 0.3 seconds With mean curculation times observed in this study lying for the most part between 10 and 15 seconds, the error is relatively small compared to any subjective methods ( $<3$ per cent).

Another simple method for determining the mean circulation time in radiocardiographic work is employed by Shipley (36). The extrapolated curves are traced on index card paper, cuttung out the enclosed area and finding the centre 


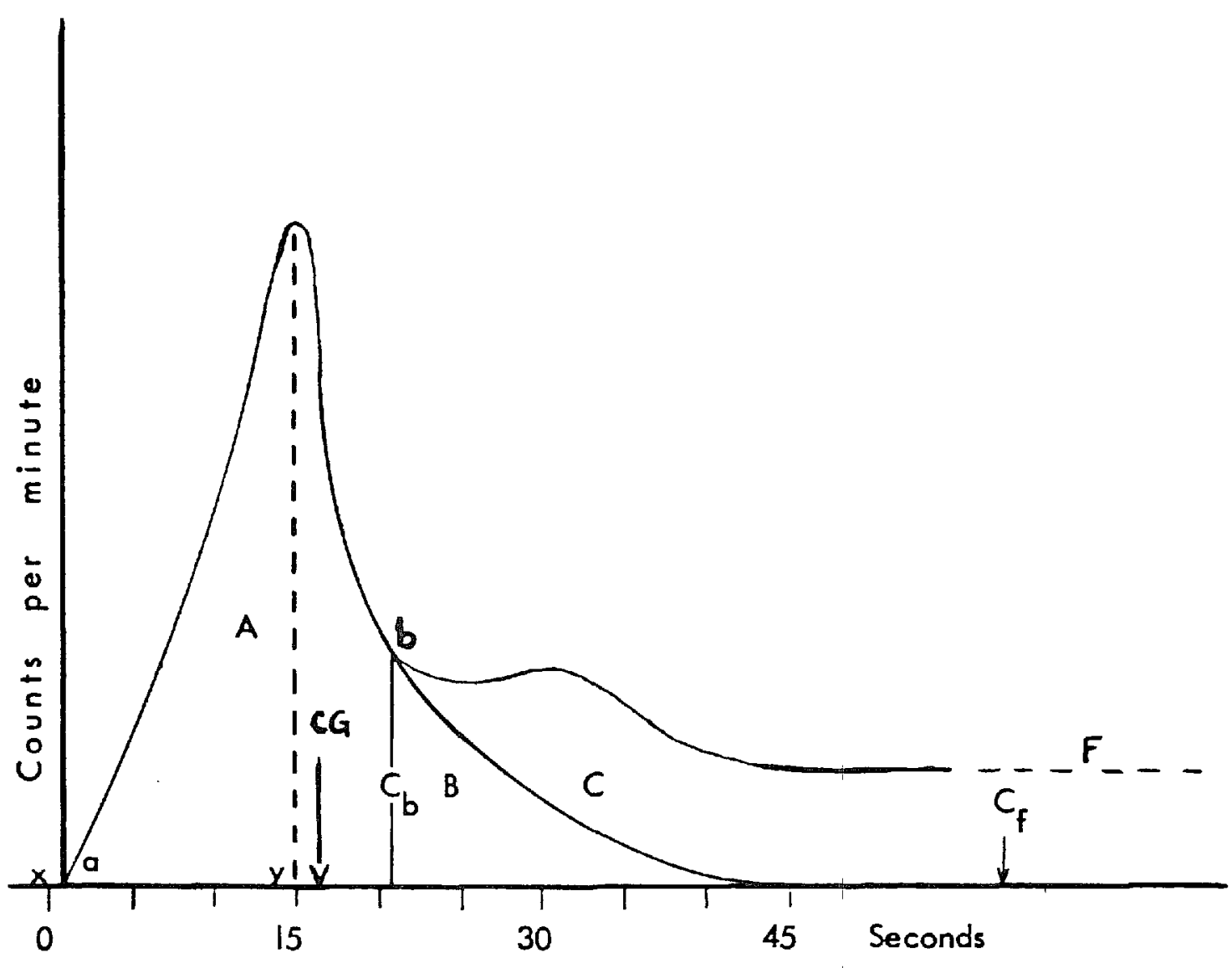

FIGURE 3. The cardiac output is derived from the area $(A+B)$ in square inches under the primary circulation tracing caused by the injected Rhisa, and extrapolated exponentially from point $b$ to the base line in order to exclude the recirculation curve $(b F)$. The final dilution of Rhisa is represented by the height $C f$ (inches). This height is multiplied by the paper speed ( 8 inches per minute), and the product divided by the area, which is measured by planimetry. The derived figure represents the cardiac output in blood volumes per minute. The latter figure is multiplied by the total blood volume, previously determined (see Fig. 4 or example). The mean circulation time is derived from the same tracing either by measuring the time $x y$ in seconds (from moment of injection to moment of peak radioactivity), or the time interval from $a$ to the projection of the centre of gravity of the primary extrapolated tracing on to the abscissa. The latter may be found empirically or mathematically (see text).

of gravity by balancing the cut-out on a knife edge. The errors by these two empirical methods ( $x y$ measure and balancing) are no greater than making multiple measurements on the curve and applying mathematics.

2. Cardiac Index $(C I)=\frac{\text { Cardiac output (litres } / \mathrm{min} .)}{\text { Body surface area }}$.

3. (a) Stroke Volume (SV) $=\frac{\text { Cardiac output (litres/min.) }}{\text { Mean pulse rate/min. }}$.

$$
\text { (b) Stroke Index }(S I)=\frac{\text { Stroke volume }}{\text { Body surface area }(B S A)} \text {. }
$$


4. Total peripheral resistance (TPR) was estimated by the over-all resistance formula of Apiera (37) in which TPR in dynes $/ \mathrm{sec} . / \mathrm{cm} .{ }^{5}$ equals

or

$$
\frac{\text { Mean arterial B.P (mmg Hg) } \times 1332}{\text { Cardiac output } \mathrm{in} \mathrm{ml} / \mathrm{sec}}
$$

$$
\frac{\text { Meeın arterial B P. }(\mathrm{mm} \mathrm{Hg}) \times 80}{\text { Cardiac output in litres }} .
$$

Differences greater than 20 per cent in the above parameters were taken as significant (38).

5. Cardiac power or left ventricular work ( $L V W)$ was estimated as recommended by Remington and Hamilton (39) according to the formula:

$L V W$ in $\mathrm{kg}$ metres $/ \mathrm{mm}=\frac{135 \times \text { cardiac output }(\mathrm{L} / \mathrm{mm}) \times \text { mean arterial B P }(\mathrm{mm} \mathrm{Hg}) .}{1000}$

This formula assumes that the specific gravity of blood is slightly greater than unity. Differences greater than 30 per cent are taken as significant.

6. The intrathoracic blood volume index (ITBVI) was derived from the formula:

Mean circulation time $\times$ cardiac output

$60 \times B S A$

A change greater than 15 per cent is considered to be significant $(38,40-44)$.

7. Oxygen consumption was measured on the respuration tracings recorded on a respirometer known as a Pullmotest ${ }^{1}$ This instrument was calıbrated with oxygen so that a one centimetre rise in the slope indicated uthlization of $300 \mathrm{ml}$. of oxygen. Paper speed on this recorder was $30 \mathrm{~mm}$. per minute. This instrument is ordinarily employed as a recording bronchospirometer and was used in this study for oxygen consumption measurements by fillng one bell with oxygen and allowing the oxygen to leak across to the other bell (filled with room air) via an electro-magnetic solenord which is activated by the tidal volume. This instrument was connected to the patient via a short piece of corrugated tubing and a well-fitting face mask selected previously (as in Fig. 1) The same fitted mask was used for each subject during all the studies.

\section{Results}

The mean restung vital signs for each subject, together with the cardiorespiratory parameters der ved from metabolic rate tables, the ventilation standards for use in artificia respiration described by Radford, and the cardiac output from the nomogram devised by Jackson, are summarized in Table I. The vital statistics, the blood volume and fractions and the mean control vital signs, together with the cardıo-respiratory parameters obtained pror to administration of the intravenous anaesthetics, are summarized in Table II. The data obtained for each subject with each drug are tabulated in Tables III to IX.

1Made by J H Becker in Delft, Holland 
TABLE I

Mean Regting Vital Signs and Phedicted Cardio-respiratory Parameters

\begin{tabular}{|c|c|c|c|c|c|c|c|c|c|}
\hline & $\mathrm{AB}$ & LC & WH & NK & DS & EB & $\mathrm{PB}$ & $A L$ & MEANS \\
\hline Systolic Blood Pressure & $\pi / 2$ & $|2|$ & 110 & 125 & 106 & 119 & 110 & IIII & 114 \\
\hline Diostolic Blood Pressure & 70 & 70 & 60 & 62 & 70 & 70 & 70 & 70 & 68 \\
\hline Pulse Pressure & 42 & 51 & 50 & 63 & 36 & 49 & 40 & 41 & 46 \\
\hline Mean Blood Pressure & 84 & 87 & 7 & 83 & 82 & 86 & 83 & 84 & 83 \\
\hline Pulso Rate & 68 & 71 & 62 & 68 & 69 & 72 & 66 & 73 & 69 \\
\hline Respiration Rate & 18 & 18 & 18 & 16 & 18 & 16 & 18 & 18 & 17.5 \\
\hline Tidal Volume** & 380 & 420 & 400 & 500 & 370 & 560 & 350 & 390 & 420 \\
\hline Minute Volume ${ }^{\star \star}$ & 6.84 & 7.85 & 7.20 & 8.00 & 6.65 & 8.98 & 6.30 & 6.82 & 7.30 \\
\hline Oxygen Consumption & 262 & 284 & 268 & 300 & 264 & 328 & 248 & 270 & 277 \\
\hline Circulation Time & 15.5 & 14.6 & 11.8 & 10.6 & 11.9 & 16.7 & 14.2 & 11.2 & 13.3 \\
\hline Cardiac Output ${ }^{+}$ & $\begin{array}{l}4.6 \\
2.55\end{array}$ & $\begin{array}{l}5.1 \\
2.52\end{array}$ & $\begin{array}{l}4.8 \\
2.60\end{array}$ & $\begin{array}{l}5.5 \\
2.62\end{array}$ & $\begin{array}{l}4.5 \\
2.42\end{array}$ & $\begin{array}{l}5.1 \\
2.22\end{array}$ & $\begin{array}{l}4.4 \\
2.63\end{array}$ & $\begin{array}{l}4.6 \\
2.35\end{array}$ & $\begin{array}{l}4.82 \\
2.48\end{array}$ \\
\hline $\begin{array}{c}\text { Stroke Volume } \\
\text { Index }\end{array}$ & $\begin{array}{l}68 \\
38\end{array}$ & $\begin{array}{l}72 \\
35\end{array}$ & $\begin{array}{l}78 \\
42\end{array}$ & $\begin{array}{l}81 \\
39\end{array}$ & $\begin{array}{l}65 \\
35\end{array}$ & $\begin{array}{l}71 \\
31\end{array}$ & $\begin{array}{l}67 \\
40\end{array}$ & $\begin{array}{l}63 \\
32\end{array}$ & $\begin{array}{l}70 \\
36\end{array}$ \\
\hline Total Peripheral Resistance & 1460 & 1370 & 1283 & 1203 & 1446 & 1346 & 1510 & 1462 & 1380 \\
\hline Left Ventricular Work & 5.2 & 6.0 & 4.9 & 6.2 & 5.0 & 5.9 & 4.9 & $5: 2$ & 5.4 \\
\hline Introthoracic Blood Volume & 1.19 & 1.24 & 0.95 & 0.98 & 0.90 & 1.42 & 1.04 & 0.86 & 1.09 \\
\hline
\end{tabular}

"Calculated from the Mayo Foundation Studies of 1936 modified to permit determination at "present age"

* * Predicted from Nomagram of Radford

+ Predicted from Nomogrom of J A ckson

Statistical analysis of these data is summarized in Tables X to XVI. The mean respiratory and haemodynamic effects of each intravenous anaesthetic are summarized in Tables XVII and XVIII respectively. Figures 4-8 show selected tracings which illustrate the typical effects observed.

The significant alteration with each drug is described below, first with regard to the acute effect, the changes from the resting and control values, and then the effects after the acute changes have passed (during "waking up" period).

Thiopental. Apnoea lasted approximately 30 seconds with the mean dose of $442 \mathrm{mg} . / \mathrm{M}^{2}$. Oxygen consumption and BMR decreased approximately 30 per cent in all subjects $(P<.01)$. This effect persisted until the subject recovered. The change was accompanied by a 16 per cent rise in pulse rate, a 17 per cent fall in the mean blood pressure and a 23 per cent fall in the venous pressure. There was no significant alteration in the mean circulation time or cardiac output. Peripheral resistance and left ventricular work showed an inconsistent decrease. These changes indicated that a substantial quantity of the circulating blood volume was probably shifted to the capillary or venous side of the circulation.

During recovery, the depression of tidal volume was compensated for adequately by a rise in the respiration rate. The pulse rate compensated for the fall in blood pressure. 


\begin{tabular}{|c|c|c|c|c|c|c|c|c|c|c|}
\hline 蛋 & $\begin{array}{l}\infty \\
2\end{array}$ & v & $\begin{array}{l}a \\
\text { m }\end{array}$ & G & $\bar{z}$ & $\begin{array}{l}\omega \\
\underline{I}\end{array}$ & $\frac{n}{n}$ & - & Subject & \multirow{27}{*}{ 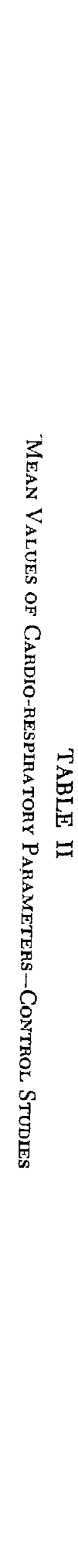 } \\
\hline & 3 & 3 & 3 & 3 & 3 & 3 & 3 & 3 & Sex & \\
\hline$N$ & $\underline{\underline{\omega}}$ & $N$ & $\tilde{\omega}$ & $\tilde{\omega}$ & $\tilde{\omega}$ & $\tilde{\omega}$ & 崩 & $N$ & Age & \\
\hline & 5 & $\frac{5}{9}$ & . & 莺 & 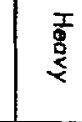 & $\frac{5}{3}$ & 曐 & 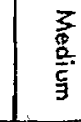 & Build & \\
\hline$\overline{\mathbf{s}}$ & $\bar{\Phi}$ & ज़ & $\underline{\underline{0}}$ & $\overline{\mathscr{G}}$ & $\overline{\mathbf{E}}$ & ज্ & $\overline{\mathscr{E}}$ & जु & Hr. cm. & \\
\hline$\alpha$ & $N$ & 8 & 엉 & $\%$ & 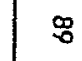 & 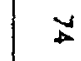 & : & 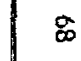 & Wr Kg. & \\
\hline $\bar{i}$ & $\bar{\delta}$ & $\bar{q}$ & $\underline{\underline{\omega}}$ & $\overline{8}$ & $\stackrel{0}{\circ}$ & 惫 & $\ddot{\underline{\theta}}$ & $\bar{\phi}$ & $\stackrel{a}{3}$ BSA & \\
\hline$\Phi$ & $\bar{N}$ & $\bar{\omega}$ & $=$ & $\bar{\infty}$ & $\bar{v}$ & $\bar{\sigma}$ & $\bar{E}$ & $\bar{\sigma}$ & $\begin{array}{l}\text { Respiration } \\
\text { Rate }\end{array}$ & \\
\hline a & $\stackrel{\infty}{0}$ & ఫ్రి & $\frac{\infty}{0}$ & पy & $\frac{n}{8}$ & 些 & छ्ठ & 总 & $\underline{3}$ Vidol & \\
\hline$\because$ & $\because$ & $\begin{array}{l}\infty \\
0 \\
0\end{array}$ & $\begin{array}{l}\infty \\
0 \\
0\end{array}$ & $\bar{i}$ & $\overline{0}$ & $\stackrel{\circ}{-}$ & $\begin{array}{l}\infty \\
\infty \\
\infty\end{array}$ & $\dot{0}$ & Mifure & \\
\hline 岕 & $\underline{y}$ & $\stackrel{w}{\mathrm{G}}$ & $\stackrel{\omega}{ \pm}$ & 岕. & 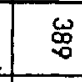 & $\frac{\omega}{\omega}$ & $\underset{W}{\omega}$ & $\frac{\omega}{\sigma}$ & 크 Oxygen. & \\
\hline$\underline{\omega}$ & 志 & 苦 & $x+$ & 志 & 屯े & \pm & ⿷્心 & $\stackrel{ \pm}{ \pm}$ & Metabolic & \\
\hline 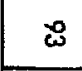 & $\bar{D}$ & 果 & 8 & 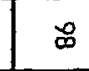 & $\bar{\sigma}$ & $\$$ & ఓ & 思 & $\begin{array}{l}\text { Mean } \\
\text { 音. Mulse Rate }\end{array}$ & \\
\hline 8 & 8 & 8 & $\infty$ & 8 & 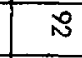 & $\infty$ & $\simeq$ & 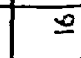 & I 3 Gloon Pressure & \\
\hline$\overline{\dot{\omega}}$ & 市 & $\bar{\sigma}$ & $i^{N}$ & $\ddot{\infty}$ & $\bar{\Sigma}_{0}$ & $\overline{\bar{\omega}}$ & $\overline{\bar{i}}$ & क & 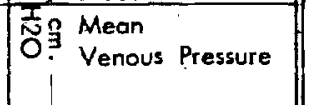 & \\
\hline ț & 느 & 点 & $\pm \overrightarrow{\tilde{\omega}}$ & $2 \frac{0}{0}$ & $\pm \stackrel{\omega}{\dot{\alpha}}$ & $\omega \stackrel{\omega}{\omega}$ & $\begin{array}{r}\omega \\
\dot{\alpha}\end{array}$ & 出 & $\begin{array}{l}\text { Plasma Volume } \\
\text { litres \& } \mathrm{ml} / \mathrm{kg}\end{array}$ & \\
\hline 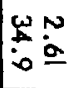 & 必望 & $\hat{N}$ & $\underline{\omega} \frac{\omega}{N}$ & ث. & ${ }_{0}^{n}$ & $\omega \stackrel{N}{N}$ & $\mu \stackrel{N}{\alpha}$ & $\omega \stackrel{\omega}{\sim}$ & $\begin{array}{l}\text { Red Cell Volume } \\
\text { litres } \$ \mathrm{~m} / \mathrm{kg}\end{array}$ & \\
\hline $\int_{i=0}^{\infty}$ & 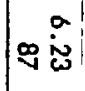 & $\bar{\delta}$ & $N$ & $\overline{9}$ & 茫 & 20 & $y \stackrel{\circ}{\infty}$ & $\ddot{E} \ddot{\omega}$ & $\begin{array}{l}\text { Total Bd. Vol. } \\
\text { litres \& } \mathrm{ml} / \mathrm{kg}\end{array}$ & \\
\hline$\doteq$ & 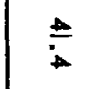 & $b_{0}^{t}$ & $\begin{array}{l}\text { A } \\
\text { in }\end{array}$ & $\stackrel{\vec{\omega}}{\dot{\omega}}$ & \pm & $\stackrel{t}{i}$ & $\stackrel{A}{:}$ & $\begin{array}{l}\text { f } \\
0\end{array}$ & Body Hcematocrth & \\
\hline $\begin{array}{l}t \\
0\end{array}$ & $\begin{array}{l}8 \\
0\end{array}$ & i & $\stackrel{t}{i}$ & $\stackrel{5}{0}$ & t5 & जे & $\stackrel{ \pm}{-}$ & in & $\begin{array}{l}\text { Venous } \\
\text { Haematocrit }\end{array}$ & \\
\hline 8 & 8 & io & 8 & 8 & 8 & 8 & 8 & 8 & $\mathrm{BH} / \mathrm{NH}$ & \\
\hline$\overline{\bar{\omega}}$ & $\overline{\bar{i}}$ & $\bar{t}$ & $\bar{v}$ & $\overline{0}_{0}$ & $\bar{\sigma}$ & $\bar{\infty}_{\infty}$ & is & $\begin{array}{l}\overline{\bar{n}} \\
\text { in }\end{array}$ & $\begin{array}{ll}\text { Mean } \\
\text { Circulation Time }\end{array}$ & \\
\hline $\begin{array}{l}\text { wa } \\
\text { in }\end{array}$ & Nu & 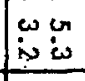 & $\begin{array}{ll}i s & 0 \\
i & \text { i } \\
\end{array}$ & {$\left[\begin{array}{l}\omega \\
v \\
v\end{array}\right.$} & $\omega v$ & $\begin{array}{ll}\omega & 0 \\
i & i\end{array}$ & Nu & $\begin{array}{l}\omega \\
i\end{array}$ & $\begin{array}{l}\text { Cardiac Output } \\
\text { and Index }\end{array}$ & \\
\hline $\begin{array}{l}\omega \% \\
\text { N }\end{array}$ & $\infty \mathcal{G}$ & $\omega$ & 10 & ผูก & 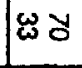 & $\Psi \sigma$ & 岕 & ज्ञ & $\begin{array}{l}\bar{B} \text { Stroke Volume } \\
\text { and Indax }\end{array}$ & \\
\hline$\overline{\overline{\bar{y}}}$ & $\overline{\bar{g}}$ & $\overline{\overline{5}}$ & $\overline{\bar{c}}$ & $\frac{6}{n}$ & 心్ & $\overline{\bar{s}}$ & $\overline{\overline{\tilde{g}}}$ & $\overline{\bar{g}}$ & $\begin{array}{l}\text { Total Peripheral } \\
\text { Resistance }\end{array}$ & \\
\hline$i$ & iv & 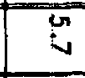 & a & int & in & $\begin{array}{c}\infty \\
\omega \\
i\end{array}$ & is & in & $\begin{array}{l}\text { 3eft Yontricular } \\
\text { Work } \mathrm{kgM} / \mathrm{min}\end{array}$ & \\
\hline 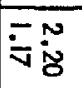 & $\dot{\infty} \overline{i 9}$ & $\overline{8}$ & $\bar{i}$ & $\vec{N}$ & $\bar{N}$ & $\overline{0}$ & is & is & $\begin{array}{l}\text { Vintrathoracic Blooo } \\
\text { Volume \& Index }\end{array}$ & \\
\hline
\end{tabular}




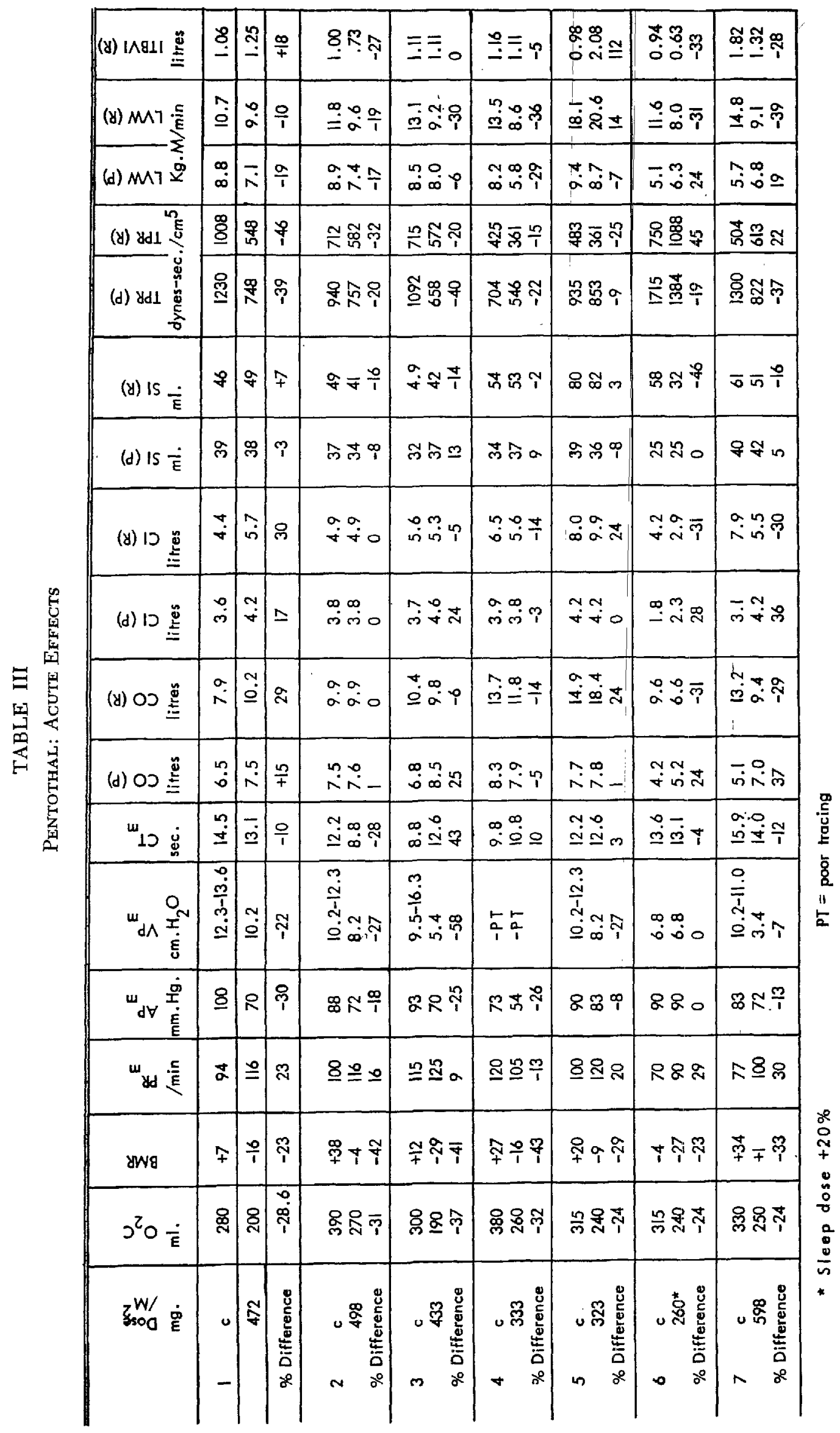




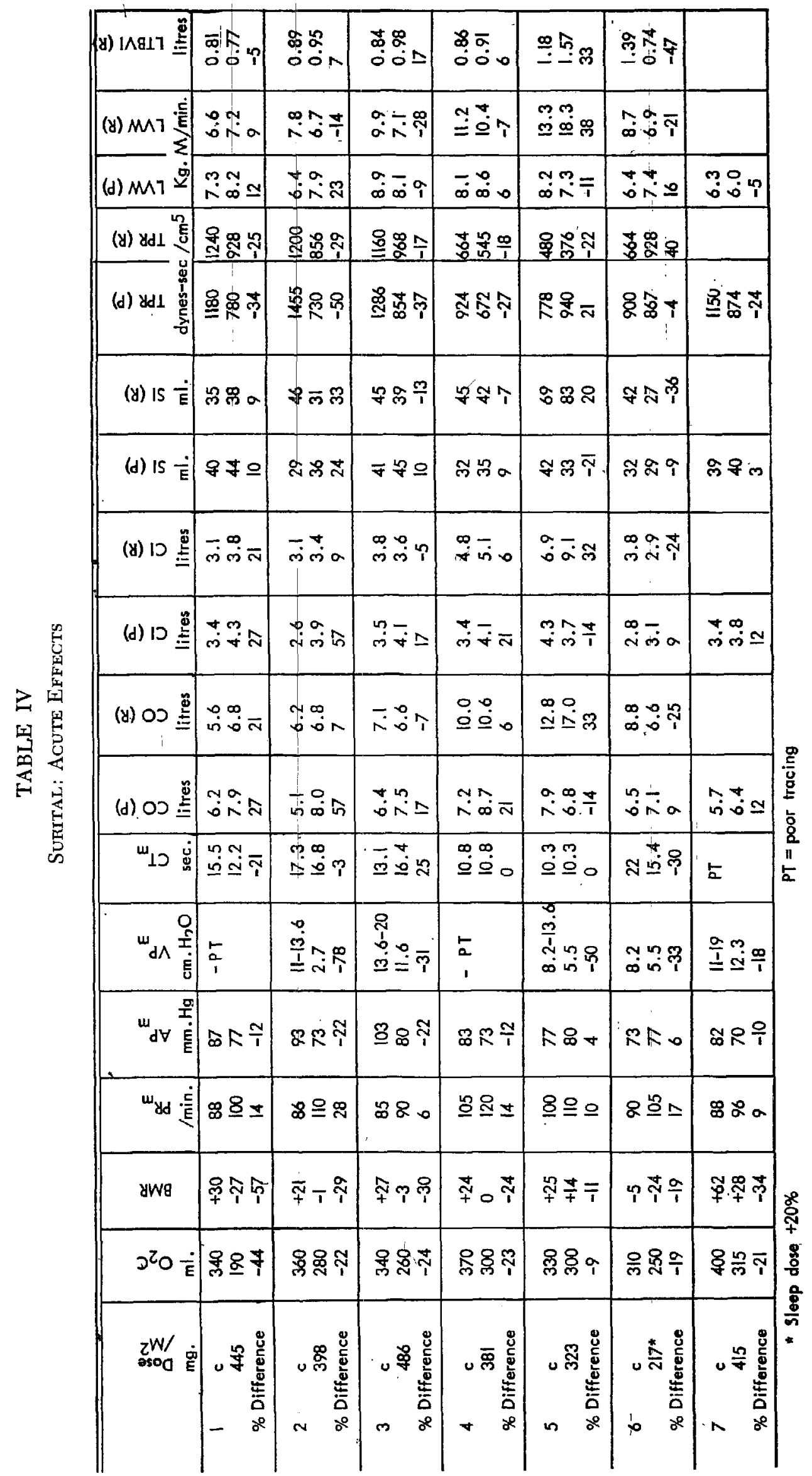




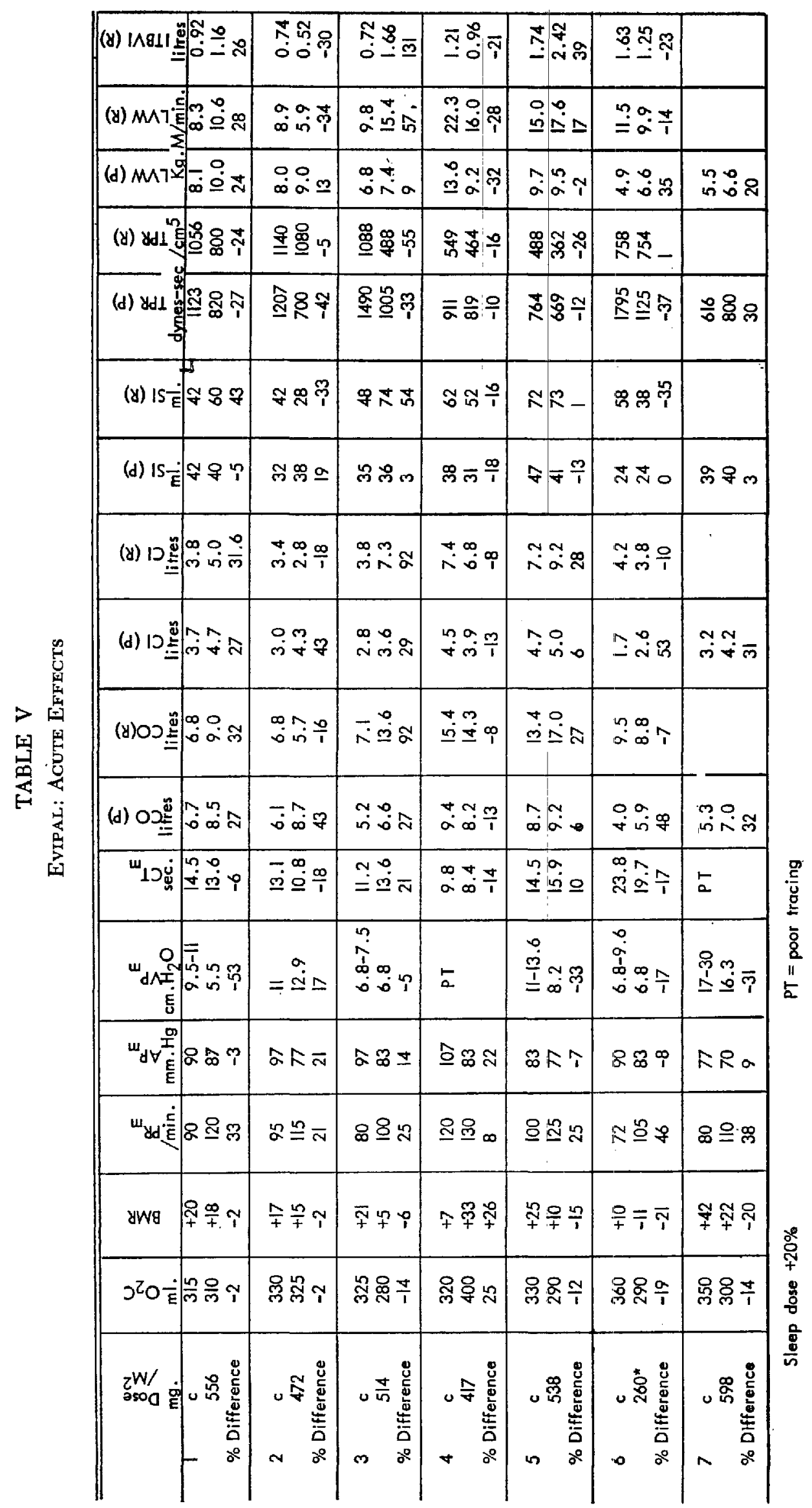




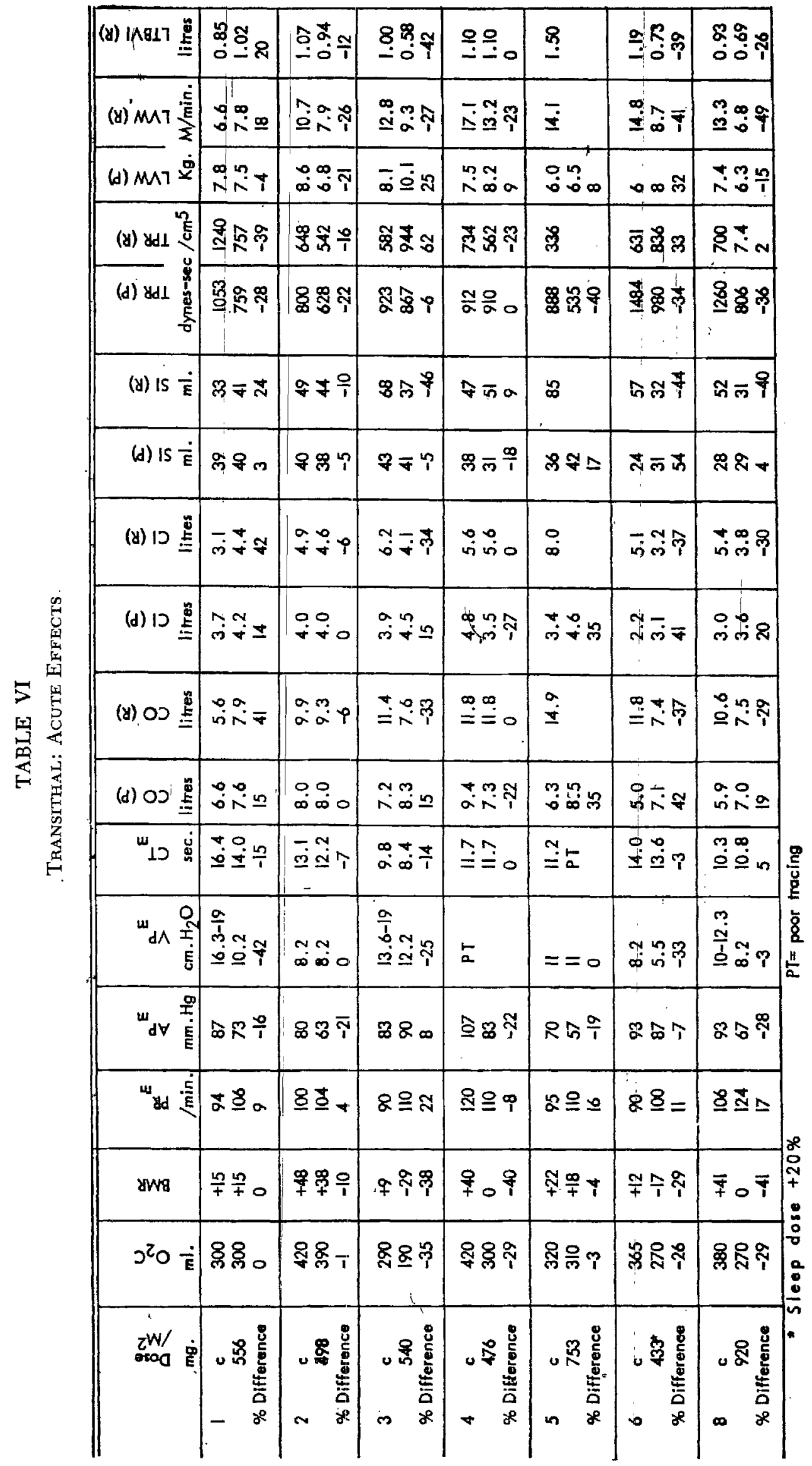




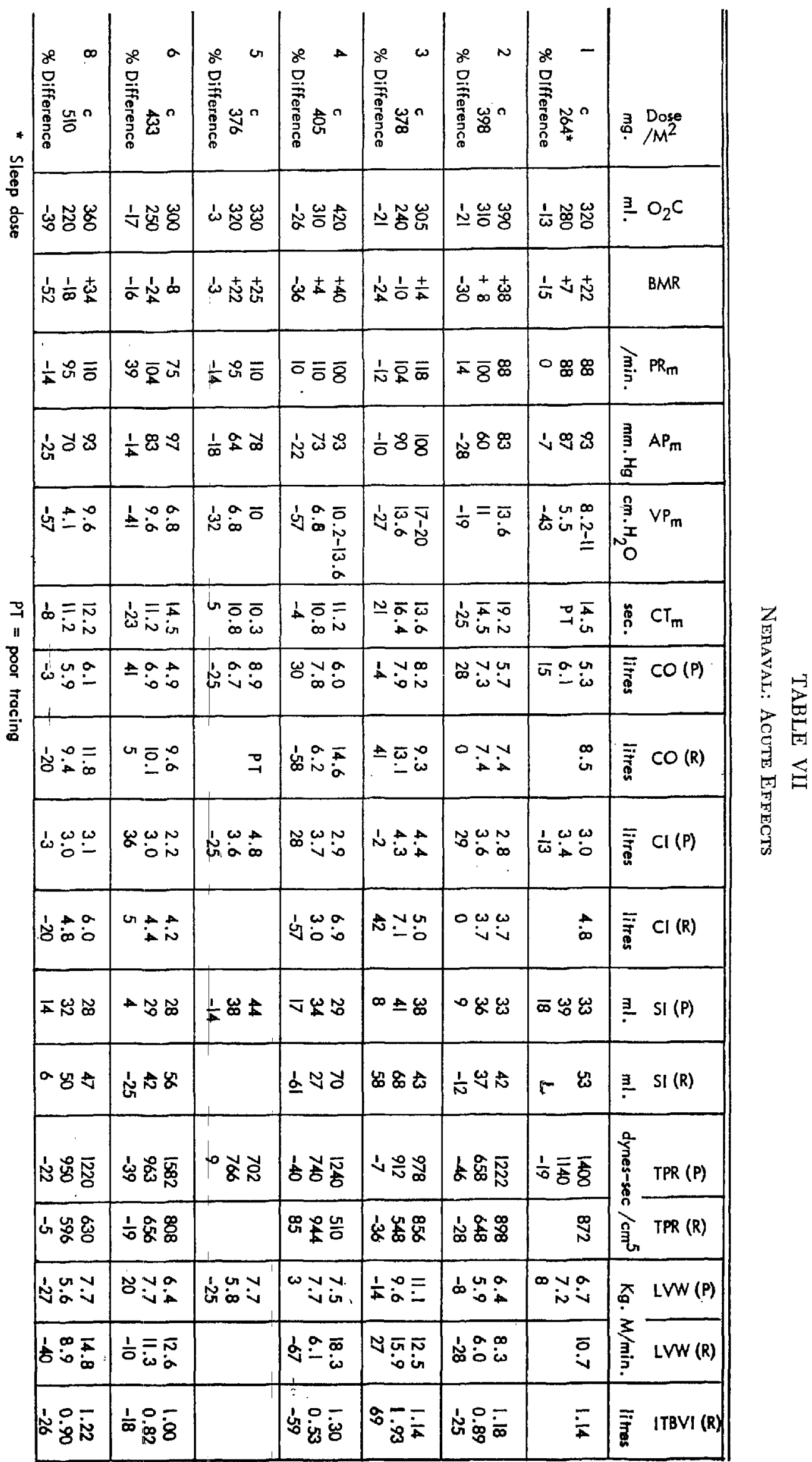




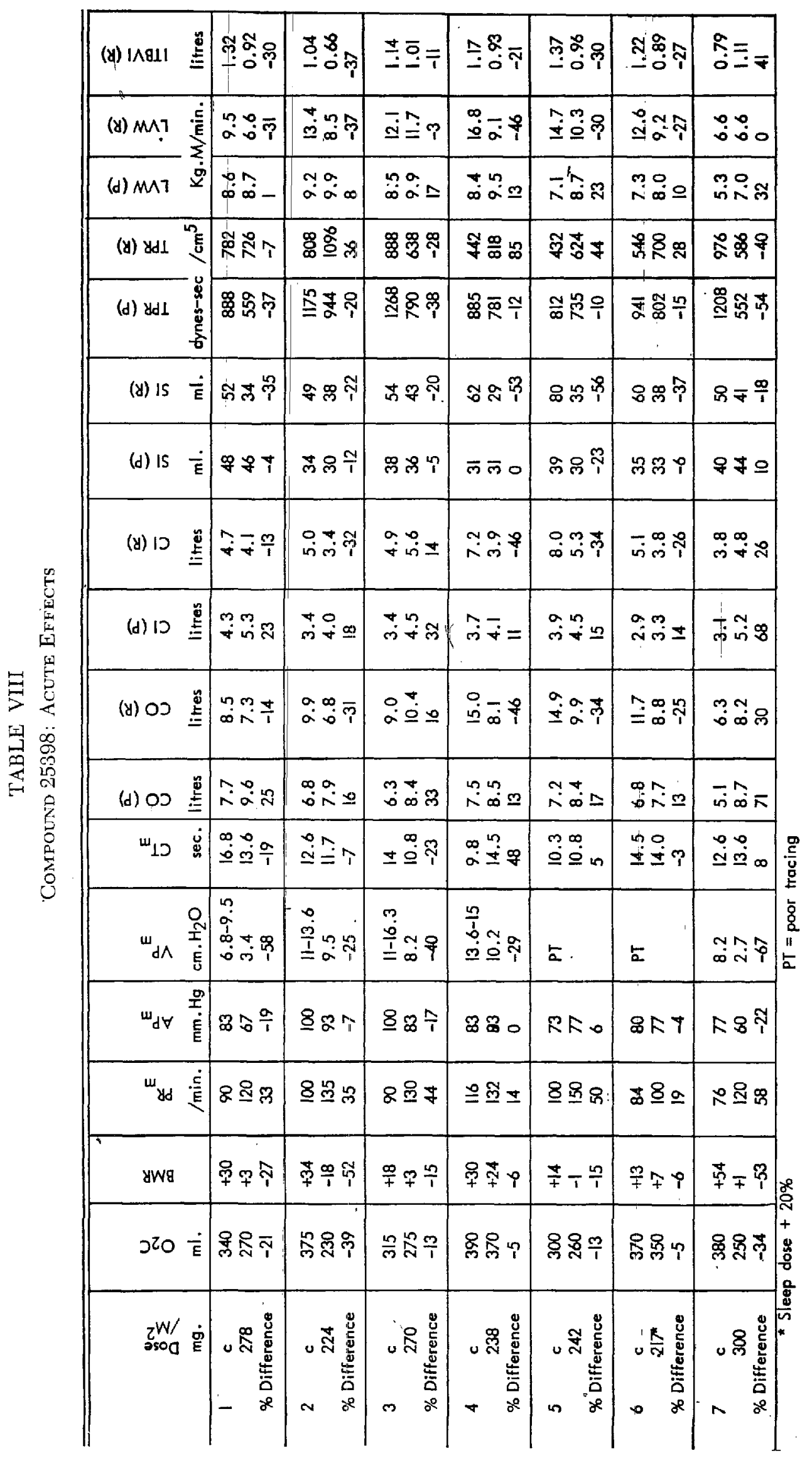




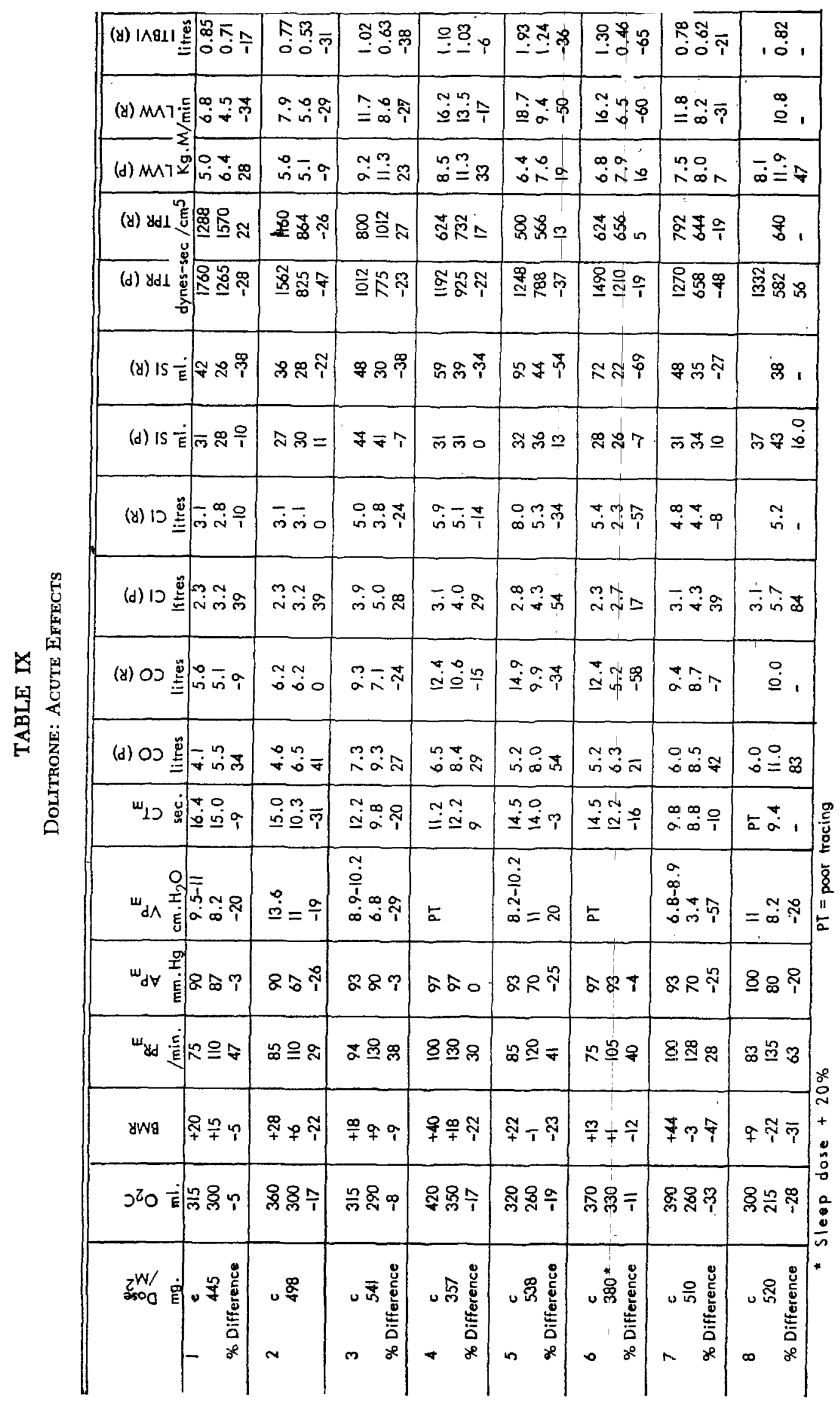



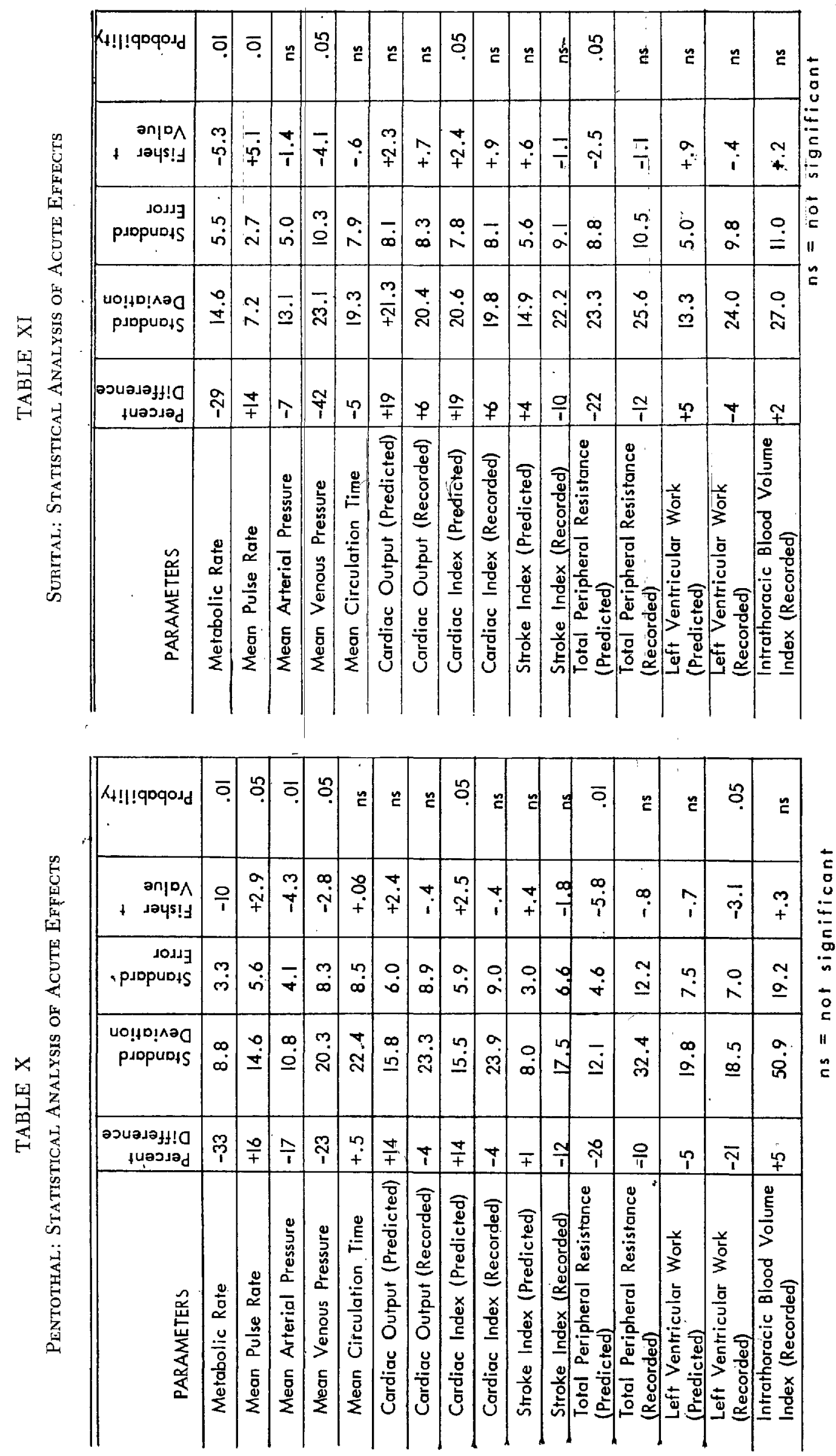


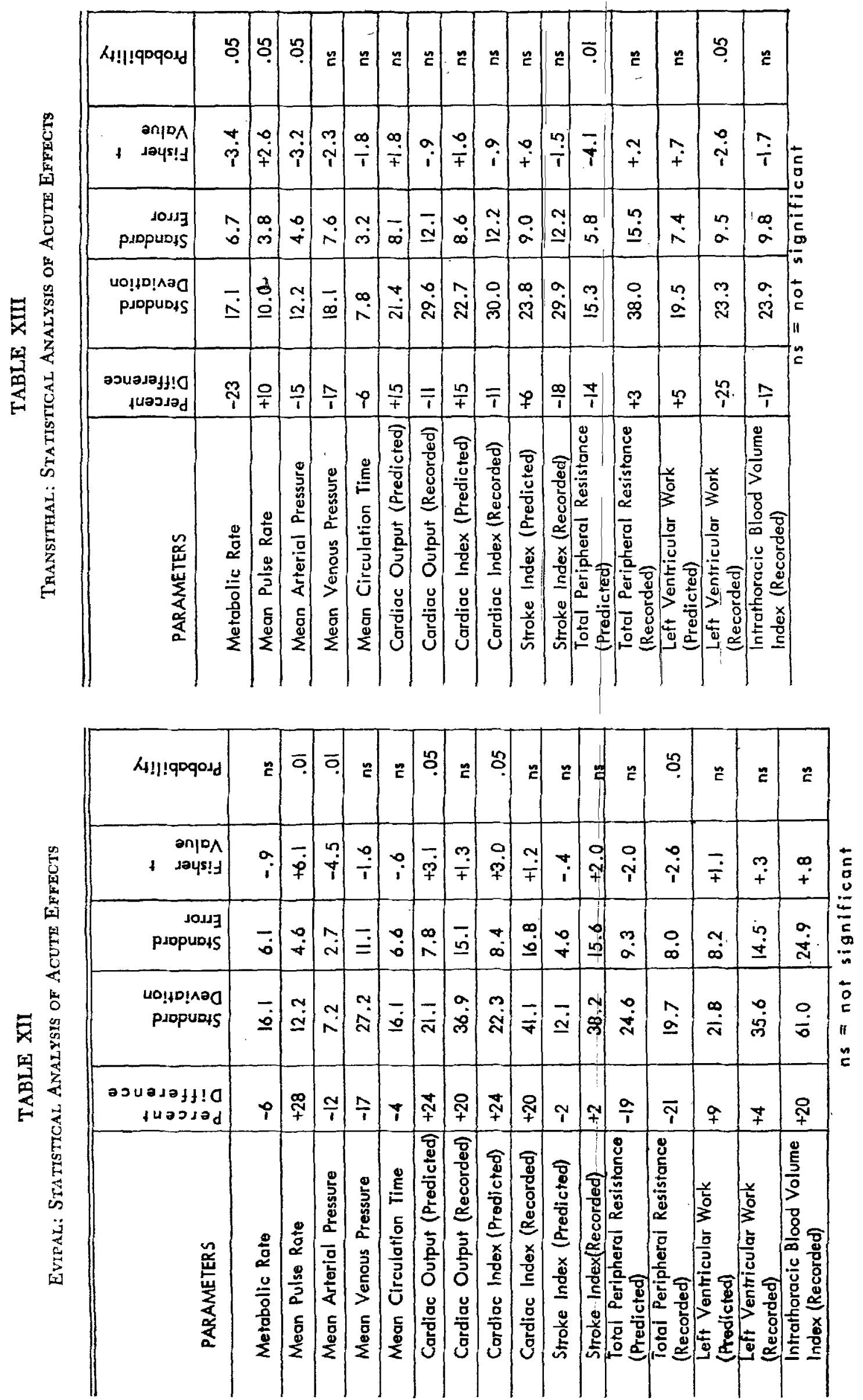



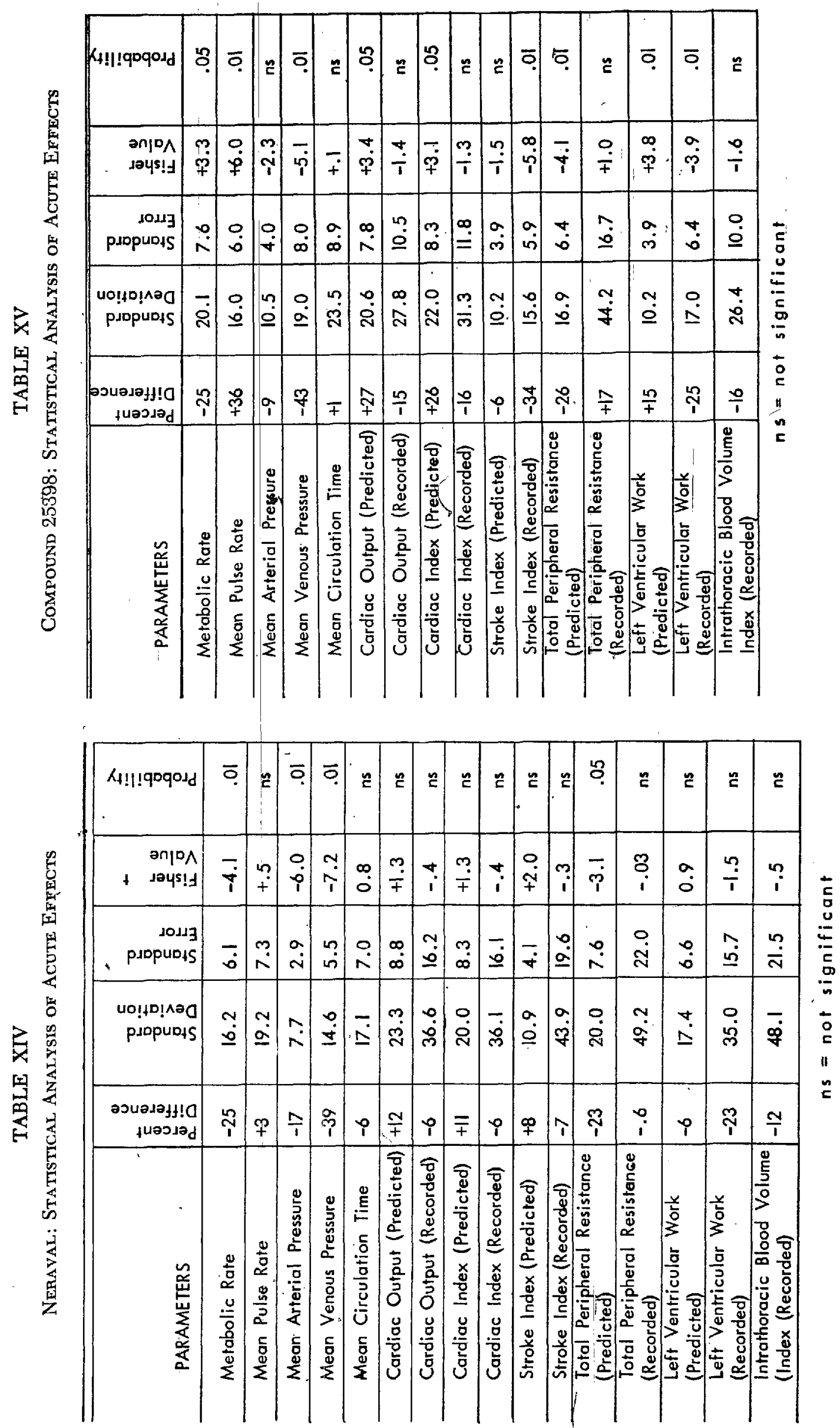
TABLE XVI

Dolitrone: Statistical Analysis of Acute Effects

\begin{tabular}{|c|c|c|c|c|c|}
\hline PARAMETERS & 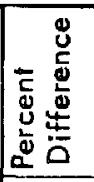 & 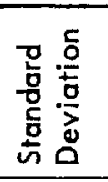 & 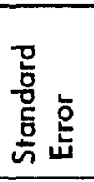 & 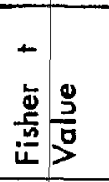 & 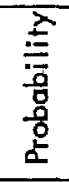 \\
\hline Metabolic Rate & -21 & 13.4 & 4.7 & -4.6 & .01 \\
\hline Mean Pulse Rate & +39 & 11.5 & 4.1 & +9.6 & .01 \\
\hline Mean Arterial Pressure & -13 & 11.5 & 4.1 & -3.2 & .05 \\
\hline Mean Venous Pressure & -22 & 24.5 & 10.0 & -2.2 & NS \\
\hline Mean Circulation Time & -11 & 12.7 & 4.8 & -2.4 & NS \\
\hline Cardiac Output (Predicted) & +41 & 19.7 & 7.0 & +5.9 & .01 \\
\hline Cardiac Output (Recorded) & -21 & 19.8 & 7.5 & -2.8 & .05 \\
\hline Cardiac Index (Predicted) & +41 & 20.3 & 7.2 & +5.7 & .01 \\
\hline Cardiac Index (Recorded) & -21 & 19.5 & 7.4 & -2.8 & .05 \\
\hline Stroke Index (Predicted) & +3 & 10.3 & 3.6 & +.9 & NS \\
\hline Stroke Index (Recorded) & -40 & 16.2 & 6.1 & -6.6 & .01 \\
\hline $\begin{array}{l}\text { Total Peripheral Resistance } \\
\text { (Predicted) }\end{array}$ & -35 & 14.0 & 4.9 & -7.2 & .01 \\
\hline $\begin{array}{l}\text { Total Peripheral Resistance } \\
\text { (Recorded) }\end{array}$ & +4 & 20.5 & 7.7 & +.5 & NS \\
\hline $\begin{array}{l}\text { Left Ventricular Work } \\
\text { (Predicted) }\end{array}$ & +20 & 16.9 & 6.0 & +3.4 & .05 \\
\hline $\begin{array}{l}\text { Left Ventricular Work } \\
\text { (Recorded) }\end{array}$ & -35 & 14.7 & 5.6 & -6.3 & .01 \\
\hline $\begin{array}{l}\text { Intrathoracic Blood Volume } \\
\text { Index (Recorded) }\end{array}$ & -30 & 19.0 & 7.1 & -4.3 & .01 \\
\hline
\end{tabular}

$n s=$ not significant

Thiamylal. The alterations in respiratory and haemodynamic parameters observed with this drug were similar to those for thiopental. The differences observed may be explained by the somewhat smaller doses of thiamylal administered, and may substantiate early clinical impressions of slightly greater potency of thiamylal with cardio-respiratory effects similar to those of thiopental.

In comparing the predicted changes with the recorded experimental data for both thiopental and thiamylal there is a depressed response by the heart, a lesser fall in total peripheral resistance and a greater fall in left ventricular work. These are indicative of cardiac depression by both thiopental and thiamylal at the dose level employed. However, the depression was not reflected in any significant fall in cardiac output.

Hexobarbital. The mean period of apnoea with this drug was 7 seconds with the mean dose of $517 \mathrm{mg}$. $/ \mathrm{M}^{2}$. There was virtually no depression of the respiration or the metabolic rate. The pulse rate rose 28 per cent while the mean arterial pressure fell 12 per cent. The mean venous pressure tended to fall, but this was not consistent. Cardiodynamic parameters changed in the 
TABLE XVII

Summary of Meain Changes in Respiration with Intravenous Anaesthetics

\begin{tabular}{|c|c|c|c|c|c|c|c|c|c|c|}
\hline & Time & $2 x$ \$le & $M^{2}$ & BMR & & $\begin{array}{r}\% \\
\text { Diff }\end{array}$ & T.V. & $\begin{array}{c}\% \\
\text { Diff }\end{array}$ & M.V. & $\begin{array}{r}\% \\
\text { Diff }\end{array}$ \\
\hline $\begin{array}{l}\text { PENTOOTHAL } \\
\text { (7 subjects) }\end{array}$ & $\begin{array}{l}R \\
C \\
A \\
T\end{array}$ & 42 & $32^{\prime \prime}$ & $\begin{array}{l}+19 \\
-33\end{array}$ & $\begin{array}{l}17.5 \\
14 \\
24\end{array}$ & $\begin{array}{l}-20 \\
+37\end{array}$ & $\begin{array}{l}720 \\
640 \\
360\end{array}$ & $\begin{array}{l}+52 \\
-14\end{array}$ & $\begin{array}{l}7.3 \\
9.0 \\
8.6\end{array}$ & $\begin{array}{l}+23 \\
+18\end{array}$ \\
\hline $\begin{array}{l}\text { SURITAL } \\
\text { (7 subjects) } \\
\end{array}$ & $\begin{array}{l}R \\
C \\
A \\
T\end{array}$ & 402 & $23^{n}$ & $\begin{array}{l}+27 \\
-29\end{array}$ & $\begin{array}{l}17.5 \\
14 \\
20\end{array}$ & $\begin{array}{l}-20 \\
+14\end{array}$ & $\begin{array}{l}420 \\
620 \\
330\end{array}$ & $\begin{array}{l}+48 \\
-21\end{array}$ & $\begin{array}{l}7.3 \\
8.7 \\
6.6\end{array}$ & $\begin{array}{l}+19 \\
-10\end{array}$ \\
\hline $\begin{array}{l}\text { EVIPAL } \\
\text { (7 subjects) }\end{array}$ & $\begin{array}{l}R \\
C \\
A \\
T\end{array}$ & 517 & $7 "$ & $\begin{array}{l}+21 \\
-6\end{array}$ & $\begin{array}{l}17.5 \\
14 \\
21\end{array}$ & $\begin{array}{l}-20 \\
+20\end{array}$ & $\begin{array}{l}420 \\
610 \\
420\end{array}$ & $\begin{array}{l}+45 \\
0 !\end{array}$ & $\begin{array}{l}7.3 \\
8.6 \\
8.8\end{array}$ & $\begin{array}{l}+18 \\
+21\end{array}$ \\
\hline $\begin{array}{l}\text { TRANSITHAL } \\
\text { (7 ubjects) }\end{array}$ & $\begin{array}{l}R \\
C \\
A \\
T\end{array}$ & 637 & $12^{14}$ & $\begin{array}{l}+28 \\
-23\end{array}$ & $\begin{array}{l}17.5 \\
20\end{array}$ & $\begin{array}{l}-14 \\
+14\end{array}$ & $\begin{array}{l}420 \\
620 \\
360\end{array}$ & $\begin{array}{l}+48 \\
-14\end{array}$ & $\begin{array}{l}7.3 \\
9.3 \\
7.2\end{array}$ & $\begin{array}{l}+27 \\
-1\end{array}$ \\
\hline $\begin{array}{l}\text { NERAVAL } \\
\text { (7 subjects) }\end{array}$ & $\begin{array}{l}\mathbf{2} \\
\mathbf{C} \\
\mathbf{A} \\
\mathbf{T}\end{array}$ & 42 & $6^{\prime \prime}$ & $\begin{array}{l}+24 \\
-25\end{array}$ & $\begin{array}{l}17.5 \\
15 \\
18\end{array}$ & $\begin{array}{l}-14 \\
+3\end{array}$ & $\begin{array}{l}420 \\
640 \\
300\end{array}$ & $\begin{array}{l}+52 \\
-21\end{array}$ & $\begin{array}{l}7.3 \\
9.6 \\
6.0\end{array}$ & $\begin{array}{l}+31 \\
-18\end{array}$ \\
\hline $\begin{array}{l}\text { LILLY } 25398 \\
\text { (7 wbjects) }\end{array}$ & $\begin{array}{l}R \\
C \\
A \\
T\end{array}$ & 273 & $82 "$ & $\begin{array}{l}+28 \\
-25\end{array}$ & $\begin{array}{l}17.5 \\
16 \\
18\end{array}$ & $\begin{array}{l}-9 \\
+3\end{array}$ & $\begin{array}{l}420 \\
570 \\
300\end{array}$ & $\begin{array}{l}+36 \\
-29\end{array}$ & $\begin{array}{l}7.3 \\
9.6 \\
6.9\end{array}$ & $\begin{array}{l}+31 \\
-5\end{array}$ \\
\hline $\begin{array}{l}\text { DOLITRONE } \\
\text { (日 subjects) }\end{array}$ & $\begin{array}{l}R \\
C \\
A \\
T\end{array}$ & 512 & $8^{\prime \prime}$ & $\begin{array}{l}+24 \\
-21\end{array}$ & $\begin{array}{l}17.5 \\
14 \\
22\end{array}$ & $\begin{array}{l}-20 \\
+26\end{array}$ & $\begin{array}{l}420 \\
640 \\
390\end{array}$ & $\begin{array}{l}+57 \\
-7\end{array}$ & $\begin{array}{l}7.3 \\
9.1 \\
8.6\end{array}$ & $\begin{array}{l}+25 \\
+18\end{array}$ \\
\hline \multicolumn{11}{|c|}{ 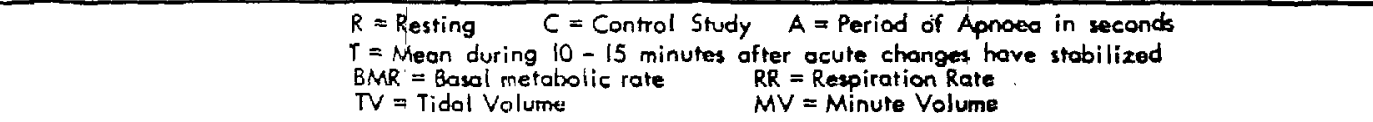 } \\
\hline
\end{tabular}

direction predicted showing a tendency to increased carcliac output and decreased total peripheral resistance without significant alteration in the left ventricular work. On the whole, hexobarbital had far less effect on either respiration or haemodynamics than thiopental and thiamylal for the corresponding anaesthetic state.

Buthalitone. The mean period of apnoea was 12 seconds with the mean dose of $637 \mathrm{mg} . / \mathrm{M}^{2}$. This was followed by a slight depression of respiration during the period of action of this drug. There was a significant fall in the metabolic rate $(23$ per cent). The pulse rate rose 10 per cent while the mean arterial pressure fell 15 per cent. This was accompanied by an inconsistent fall in mean venous pressure. Cardiodynamic alterations were inconsistent but tended towards a slight fall in cardiac output without any significant change in the total peripheral resistance. However, the left ventricular work decreased by 25 per cent. In the parameters studied, this drug has an effect which is not unlike that of hexobarbital, except for the greater apparent effect on the heart.

Methitural. Although the potency of this drug is similar to that of thiopental and one-third greater than that of buthalitone, according to the dose adminis- 


\section{TABLE XVIII}

Summary of Mean Haemodynamic Effects with Intravenous Anaesthetics

\begin{tabular}{|c|c|c|c|c|c|c|c|c|c|c|c|}
\hline $\begin{array}{c}\text { DRUG } \\
\text { PENTOTHAL } \\
\text { (7 mbjects) }\end{array}$ & $\begin{array}{l}R \\
C \\
A \\
T\end{array}$ & \begin{tabular}{|c|} 
SBP \\
114 \\
119 \\
97 \\
98 \\
\end{tabular} & $\begin{array}{c}\% \\
\text { Diff. } \\
+4.4 \\
-15 \\
-14\end{array}$ & $\begin{array}{l}\text { DBP } \\
68 \\
72 \\
62 \\
60\end{array}$ & $\begin{array}{c}\% \\
\text { DIfF. } \\
+5.9 \\
-8.8 \\
-17.7\end{array}$ & $\begin{array}{l}8 \\
83 \\
82 \\
74 \\
73\end{array}$ & $\begin{array}{c}\% \\
\text { Diff. } \\
-1.2 \\
-10.8 \\
-12\end{array}$ & $\begin{array}{l}P P_{m} \\
46 \\
47 \\
35 \\
40\end{array}$ & $\begin{array}{c}\% \\
\text { Diff. } \\
+2.2 \\
-24 \\
-13\end{array}$ & $\begin{array}{c}R_{m} \\
69 \\
85 \\
111 \\
96\end{array}$ & $\begin{array}{c}\% \\
\text { Diff. } \\
\pm 23 \\
+61 \\
+39\end{array}$ \\
\hline $\begin{array}{l}\text { SLRITAL } \\
\text { (7 wbjects) }\end{array}$ & $\begin{array}{l}R \\
C \\
A \\
T\end{array}$ & $\begin{array}{l}114 \\
121 \\
103 \\
103\end{array}$ & $\begin{array}{l}+6.1 \\
-9.7 \\
-9.7\end{array}$ & $\begin{array}{l}68 \\
72 \\
62 \\
63\end{array}$ & $\begin{array}{l}+5.9 \\
-8.8 \\
-7.4\end{array}$ & $\begin{array}{l}83 \\
88 \\
76 \\
76\end{array}$ & $\begin{array}{l}+6 \\
-8.4 \\
-8.4\end{array}$ & $\begin{array}{l}46 \\
49 \\
4 ! \\
40\end{array}$ & $\begin{array}{l}+6.5 \\
-11 \\
-13\end{array}$ & $\begin{array}{l}69 \\
93 \\
104 \\
103\end{array}$ & $\begin{array}{l}+35 \\
+51 \\
+49\end{array}$ \\
\hline $\begin{array}{l}\text { EVIPAL } \\
\text { (7 subjects) }\end{array}$ & $\begin{array}{l}R \\
C \\
A \\
T\end{array}$ & $\begin{array}{l}114 \\
137 \\
107 \\
115\end{array}$ & $\begin{array}{l}+20.2 \\
-6.1 \\
+1\end{array}$ & $\begin{array}{l}68 \\
75 \\
66 \\
63\end{array}$ & $\begin{array}{l}+10.3 \\
-3 \\
-7.4\end{array}$ & $\begin{array}{l}83 \\
96 \\
80 \\
80\end{array}$ & $\begin{array}{r} \\
+15.7 \\
-3.6 \\
-3.6\end{array}$ & $\begin{array}{l}46 \\
62 \\
41 \\
52\end{array}$ & $\begin{array}{l}+35 \\
-11 \\
+13\end{array}$ & $\begin{array}{l}69 \\
95 \\
115 \\
116\end{array}$ & $\begin{array}{l}+37 \\
+66 \\
+68\end{array}$ \\
\hline $\begin{array}{l}\text { TRANSITHAL } \\
\text { (7 subjectr) }\end{array}$ & $\begin{array}{l}\cdot R \\
C \\
A \\
T\end{array}$ & $\begin{array}{l}114 \\
126 \\
102 \\
105\end{array}$ & $\begin{array}{l}+10.5 \\
-9.7 \\
-8.6\end{array}$ & $\begin{array}{l}68 \\
68 \\
61 \\
61\end{array}$ & $\begin{array}{c}0 \\
-10.3 \\
-10.3\end{array}$ & $\begin{array}{l}83 \\
87 \\
75 \\
76\end{array}$ & $\begin{array}{l}+4.8 \\
-9.7 \\
-8.4\end{array}$ & $\begin{array}{l}46 \\
58 \\
42 \\
44\end{array}$ & $\begin{array}{l}+26 \\
-8.7 \\
-4.3\end{array}$ & $\begin{array}{c}69 \\
100 \\
109 \\
109\end{array}$ & $\begin{array}{r}+45 \\
+58 \\
+58\end{array}$ \\
\hline $\begin{array}{l}\text { INERAVAL } \\
\text { (7 ubjects) }\end{array}$ & $\begin{array}{l}R \\
C \\
A \\
T\end{array}$ & $\begin{array}{l}114 \\
119 \\
102 \\
103\end{array}$ & $\begin{array}{l}+4.4 \\
-10.5 \\
-10\end{array}$ & $\begin{array}{l}68 \\
75 \\
61 \\
62\end{array}$ & $\begin{array}{l}+10.3 \\
-10.3 \\
-10.3\end{array}$ & $\begin{array}{l}83 \\
90 \\
75 \\
75\end{array}$ & $\begin{array}{l}+8.4 \\
-9.7 \\
-9.7\end{array}$ & $\begin{array}{l}46 \\
44 \\
41 \\
41\end{array}$ & $\begin{array}{l}-4.3 \\
-11 \\
-11\end{array}$ & $\begin{array}{c}69 \\
88 \\
100 \\
87\end{array}$ & $\begin{array}{r}: \\
+28 \\
+45 \\
+26\end{array}$ \\
\hline $\begin{array}{l}\text { Lilly } 25398 \\
\text { (7 subjøets) }\end{array}$ & $\begin{array}{l}R \\
C \\
A \\
T\end{array}$ & $\begin{array}{l}114 \\
124 \\
103 \\
105\end{array}$ & $\begin{array}{l}+8.8 \\
-9.7 \\
-8.6\end{array}$ & $\begin{array}{l}68 \\
70 \\
64 \\
72\end{array}$ & $\begin{array}{l}+3 \\
-5.9 \\
+5.9\end{array}$ & $\begin{array}{l}83 \\
88 \\
77 \\
83\end{array}$ & $\begin{array}{c}+6 \\
-7.2 \\
0\end{array}$ & $\begin{array}{l}46 \\
54 \\
39 \\
33\end{array}$ & $\begin{array}{l}+18 \\
-15 \\
-28\end{array}$ & $\begin{array}{c}69 \\
99 \\
126 \\
129\end{array}$ & $\begin{array}{l}+43 \\
+\infty 3 \\
+87\end{array}$ \\
\hline $\begin{array}{c}\text { DOLITRONE } \\
\text { (ubjech) }\end{array}$ & $\begin{array}{l}R \\
C \\
A \\
T\end{array}$ & $\begin{array}{l}114 \\
121 \\
105 \\
116\end{array}$ & $\begin{array}{l}+6.1 \\
-8.6 \\
+1.8\end{array}$ & $\begin{array}{l}68 \\
78 \\
70 \\
80\end{array}$ & $\begin{array}{l}+14.7 \\
+3 \\
+17.7\end{array}$ & $\begin{array}{l}83 \\
92 \\
82 \\
92\end{array}$ & $\begin{array}{l}+10.8 \\
-1.2 \\
+10.8\end{array}$ & $\begin{array}{l}46 \\
43 \\
35 \\
36\end{array}$ & $\begin{array}{l}-6.5 \\
-24 \\
-22\end{array}$ & $\begin{array}{c}69 \\
88 \\
121 \\
111\end{array}$ & $\begin{array}{l}+28 \\
+75 \\
+61\end{array}$ \\
\hline & & & $\begin{array}{l}\text { control } \\
\text { acuto } \\
\text { mean d }\end{array}$ & Jurir & $\begin{array}{l}S B P= \\
D B P= \\
B P_{m} \\
-15\end{array}$ & & Bloo & & $\begin{array}{l}\text { sure } p \\
\text { essure } p \\
\text { ere }\end{array}$ & & $\begin{array}{l}\text { Pulse Pressure } \\
\text { n Pulse Rate } \\
\text { bilized. }\end{array}$ \\
\hline
\end{tabular}

tered (432 mg./ $\mathrm{M}^{2}$ ), all changes observed in these experiments were in the same direction and of the same order of magnitude;as seen with buthalitone.

Compound 25398. The mean period of apnoea was 82 seconds with mean dose of $273 \mathrm{mg} . / \mathrm{M}^{2}$. Metabolic rate fell 25 per cent. Pulse rate rose 36 per cent 


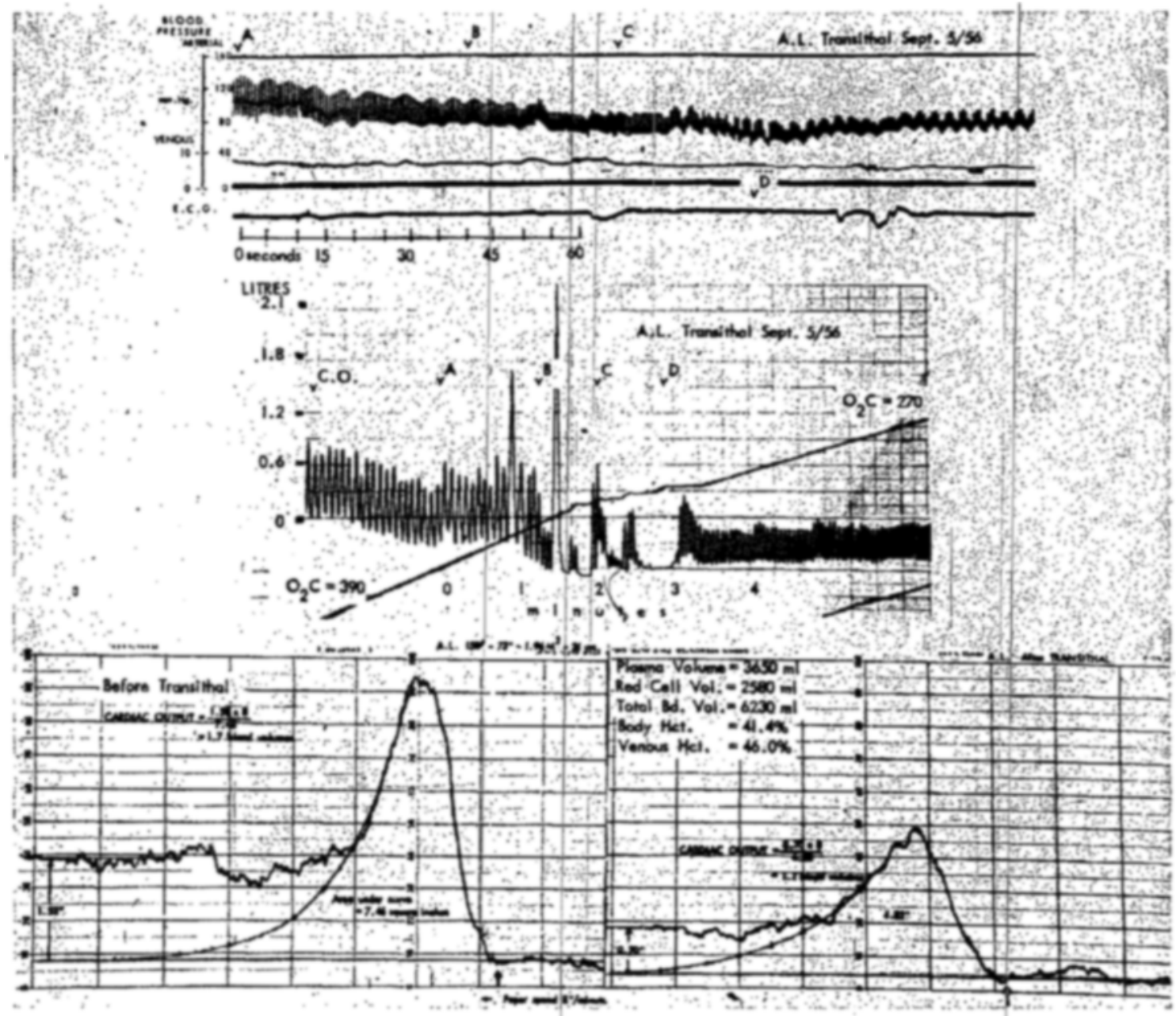

FIGURE $_{*}$ 4. Tracing from a typical experiment showing simultaneous determinations of haemodynamic and respiratory parameters: upper shows arterial and venous pressure (ECG is too compressed in copy); middle shows respiration and oxygen consumption; lower shows cardiac output. " $\mathrm{A}$ " represents beginning of injection of drug. " $\mathrm{B}$ " represents beginning of injection of second sleep dose and " $\mathrm{C}$ " is end of injection. " $\mathrm{C} O$ " and " $D$ " represent the control and test injections of Rhisa for the cardiac output estimation. On the respiration tracing the sloping line represents the oxygen consumption, which is noted before and after the injections. Method of computing the cardiac output is shown on the recording from the count-rate meter and Varian recorder.

while mean arterial blood pressure fell 9 per cent. There was a consistent fall in the mean venous pressure of 43 per cent. Cardiac output fell inconsistently to 15 per cent below resting levels while total peripherai resistance rose inconsistently to 17 per cent above resting levels. There was a 25 per cent fall in left ventricular work. These observed changes place this compound in the same class as thiopental and thiamylal, but with greater potency and greater depression of cardio-respiratory dynamics.

Dolitrone. The mean period of apnoea lasted 8 seconds with the mean dose of $512 \mathrm{mg} . / \mathrm{M}^{2}$, while metabolism was reduced 21 per cent. Pulse rate rose 39 per cent; mean arterial pressure fell 13 per cent. There was an inconsistent fall in the mean venous pressure of 22 per cent. There was a consistent fall in the cardiac output of approximately 20 per cent while the total peripheral 


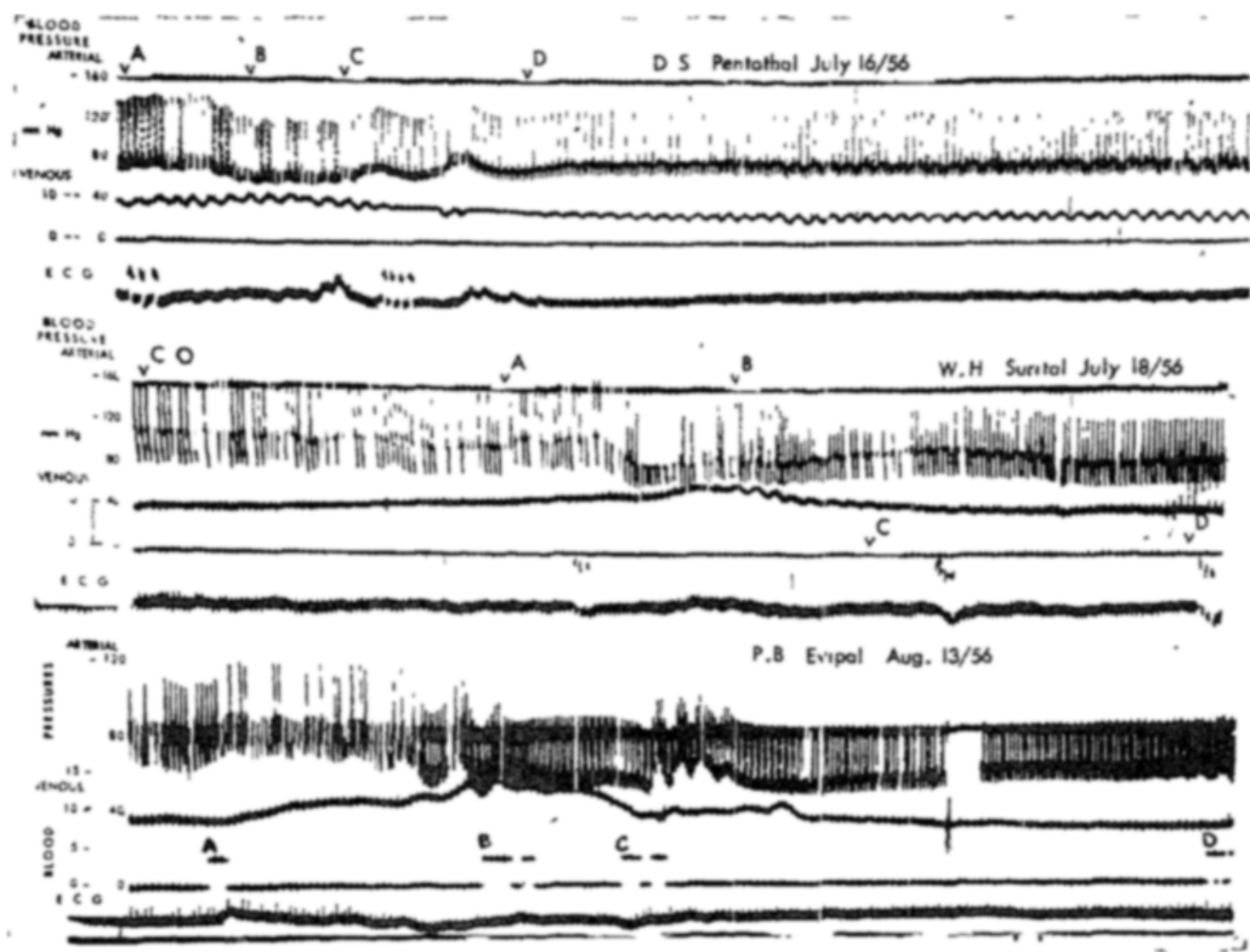

Figure 5. Haemodynamic response to thopental, thiamylal and hexobarbital by a double dose Note the rise in venous pressure preceding the start of injection, and then a fall to below the control level Note the persistent depression in arterial blood pressure after the initral acute fall, which is greatest with hexobarbital and least with thiopental

resistance tended to ruse slightly Left ventricular work decreased by 35 per cent.

The potency of this drug approximated that of hexobarbital very closely. Respiratory effects of this drug were also very similar to those seen with hexobarbital and buthaliton However, the cardıodynamic depression by this drug was much greater than with both hexobarbital and buthalitone

\section{Discussion}

\section{Blood Volume and Cardiac Output}

The determination of total blood volume and cardiac output are critical values in this study It is essential therefore to elaborate on their accuracy It has been shown that "complete mixing" of an injected tracer substance such as Rihsa for determination of plasma volume occurs in the range of 4 to 15 minutes and that mixing tmes are discrete from the disappearance slope in most subjects (45, 46,47 ) In this study a single 15 -minute sample was taken in these normal subjects and was assumed to have complete mixing so that plasma volume would be calculated accurately. The determination of red cell volume by radioactive chromium is also a highly accurate determination according to Sterling and Gray 


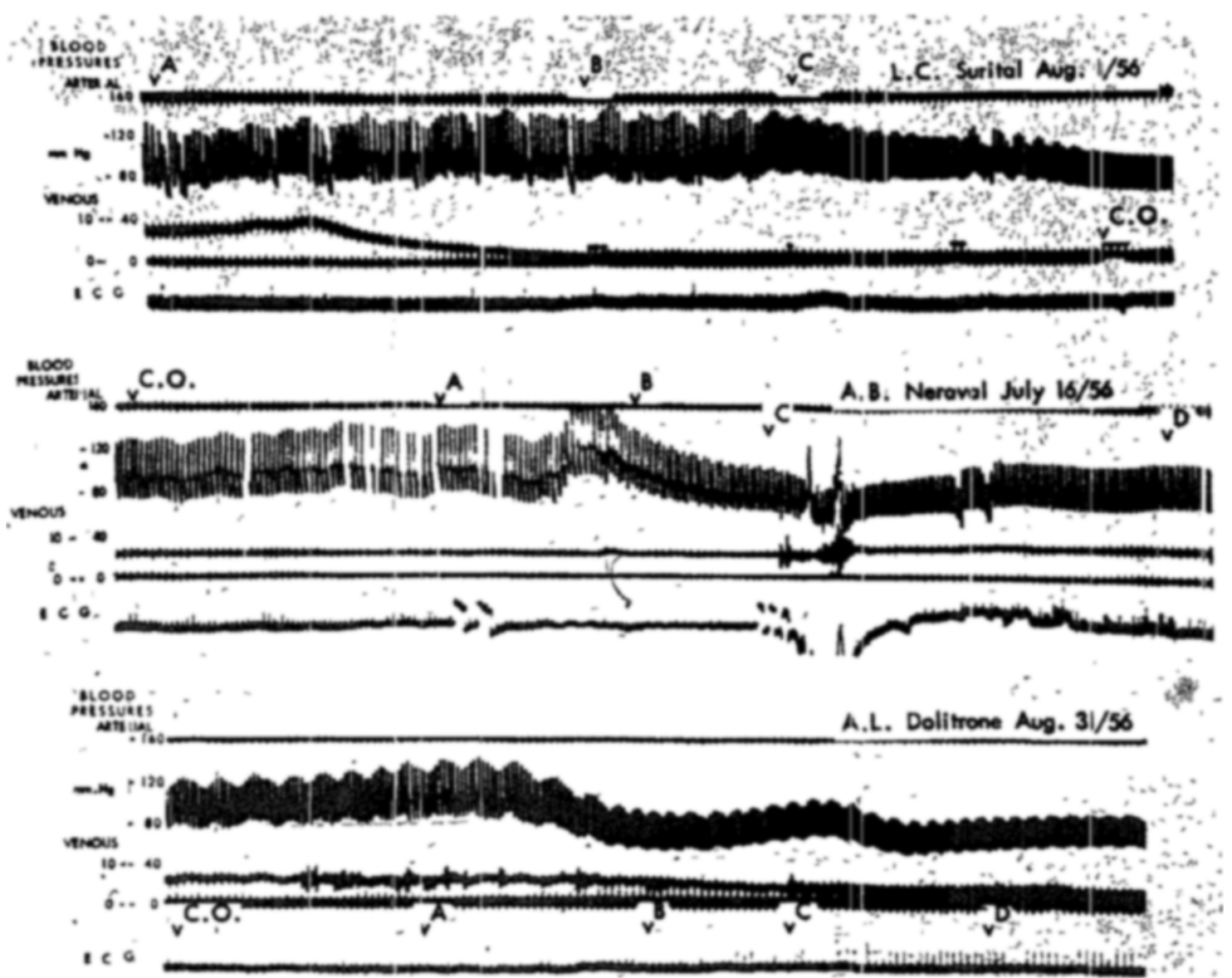

Figure 6 Haemodynamic response to thiamylal, methitural and Dolitrone. Note depression of arterkal pressure for all three and marked fall in venous pressure with thramylal and Dolitrone

(22). The simultaneous measurement of plasma volume, red cell volume and venous haematocrit in the eight normal subjects in this study showed an almost constant 'ratio of body haematocrit to the venous haematocrit $(0.90)$. These observations agree very closely with those of Hicks and associates (48) and those found in another study (27).

The determination of cardiac output by the injection method is based on the measurement of the varying concentration of the injected material as it passes through the heart. If a small volume of highly concentrated material is injected into the inflow tract, the average dilution of the material coming out from the heart will be a direct indication of the volume passing through the heart and causing this dilution. The measurement of the change of concentration durng a definite interval permits the output to be expressed as volume of flow per unit time, as measured on a recorded cardiac dilution curve (45).

The main advantage of using iodine ${ }^{131}$ tagged serum albumin is that this concentration may be determined by gamma-ray assay. A contnuous sampling of concentration without sample preparation may then be made, since the absorption in the blood or thssue is negligible. This obviates the laborious system of withdrawing arterial blood samples and assaying the samples, and makes 


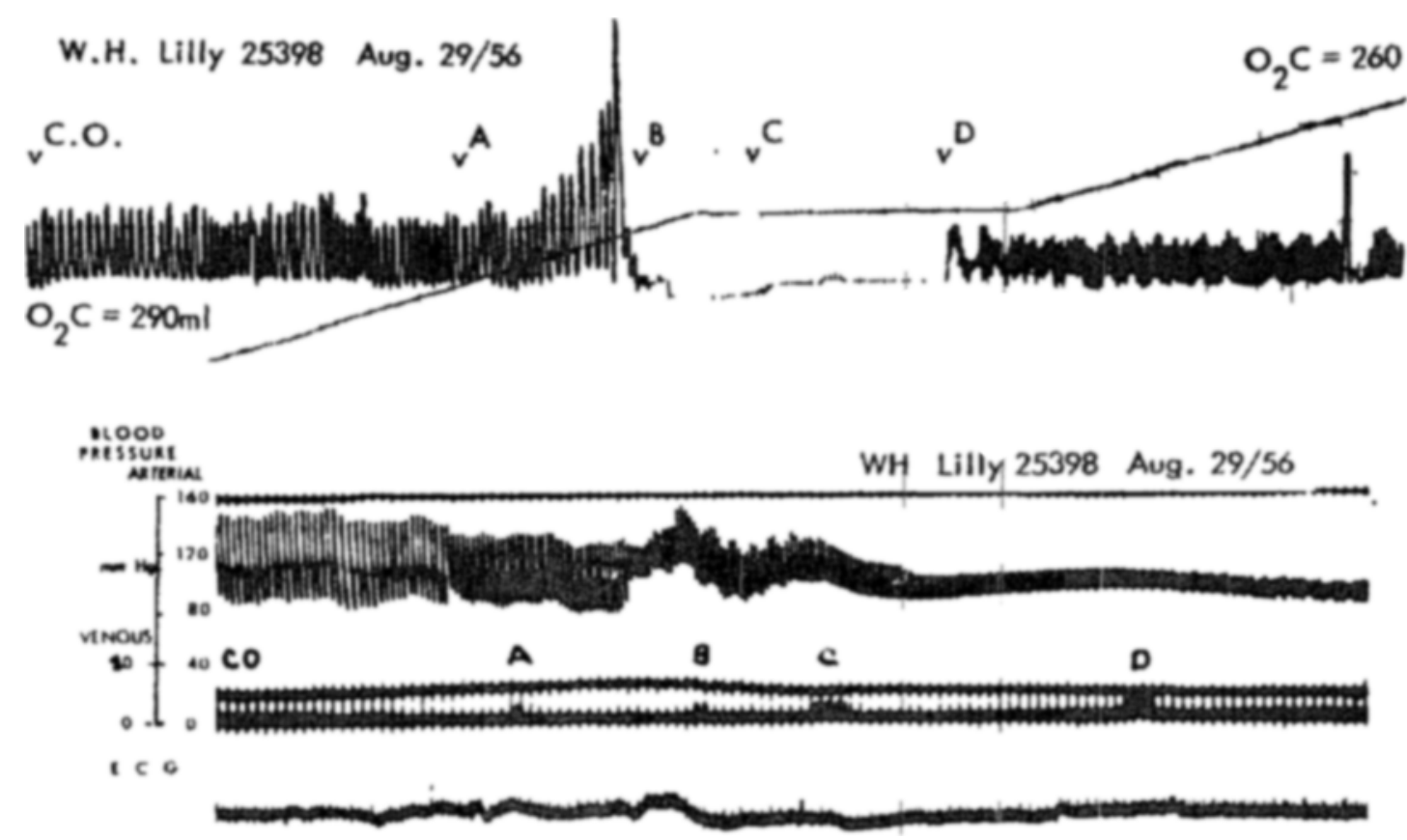

Figure 7. Respiratory and haemodynamic response to Cpd 25398 (Lilly) (double the sleep dose administered in a $2.5 \%$ solution). Note long period of apnoea and marked depression of respiration, arterial blood pressure and pulse pressure accompanied by marked tachypnoea and tachycardia.

accurate timing of the dilution curve more easily obtainable. Since all critical time measurements were made primarily within sixty seconds (including the time from the moment injection started), and the loss of serum albumin from the vascular system is not more than about 3 per cent for the first ten minutes following injection, the method is entirely satisfactory for precise measurement of the period being studied and has a high order of accuracy compared to the dye injection method $(49,50)$.

Because the injected material is diluted very rapidly, it is necessary to employ a detector of the highest sensitivity. A highly collimated crystal detector (scintillation counter) with high sensitivity to gamma radiation is therefore essential for these estimations. Such a detector must be directed over the area of the chest where the least interference from other blood flows is located, namely the first left interspace of the chest, adjacent to the sternum (51). The dilution curve or the change in concentration of the diluted material in the blood is recorded by a scaler and counting rate-computer combination and inscribed automatically by a direct writing recorder. A fast response time-counting rate meter is a'so satisfactory, but the scaler-computer mbination eliminates the memory effect of the rate meter and allows a lower counting rate and dose to be used. Successful repeated measurements can be made with 25 to 75 microcuries $(52,53,54)$.

The dilution curve has the same general form as that seen with Evans Blue dye. There is characteristically a rapid initial rise as the bulk of the radioactivity passes the counter site, followed by a steep fall to a partially super- 


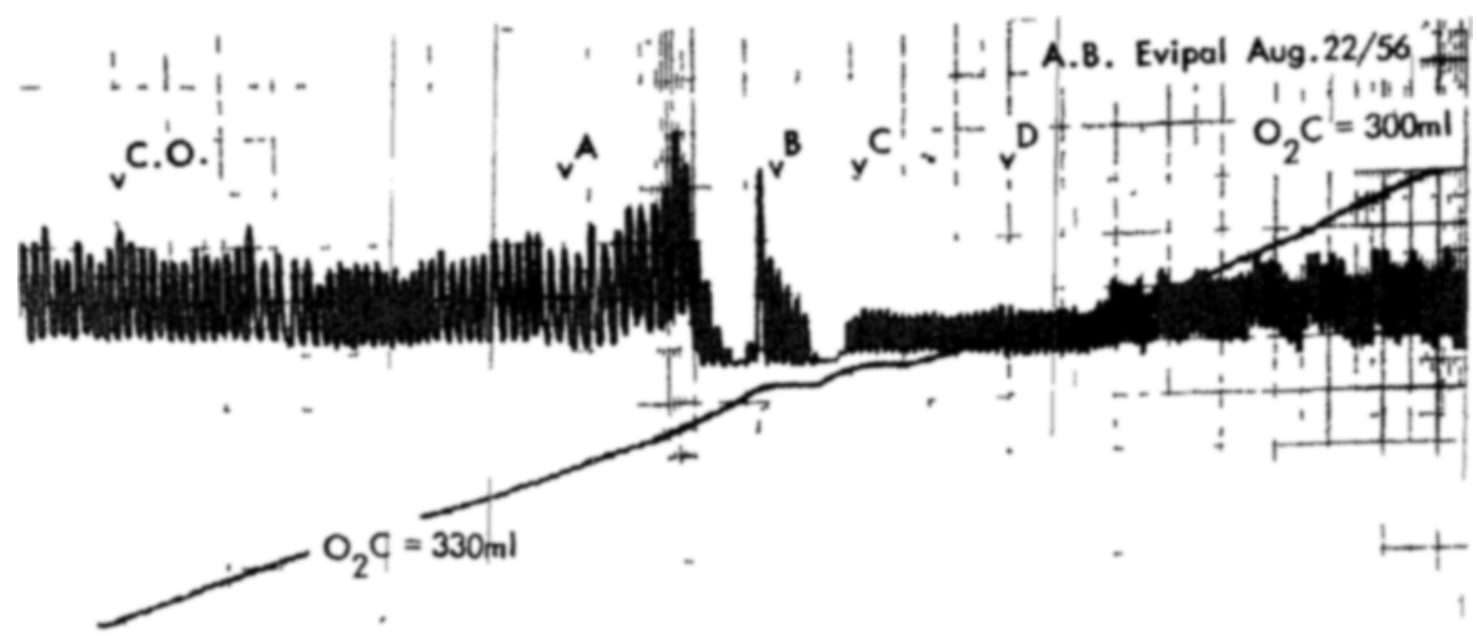

\section{E.B. Dolitrone July $30 / 56$}

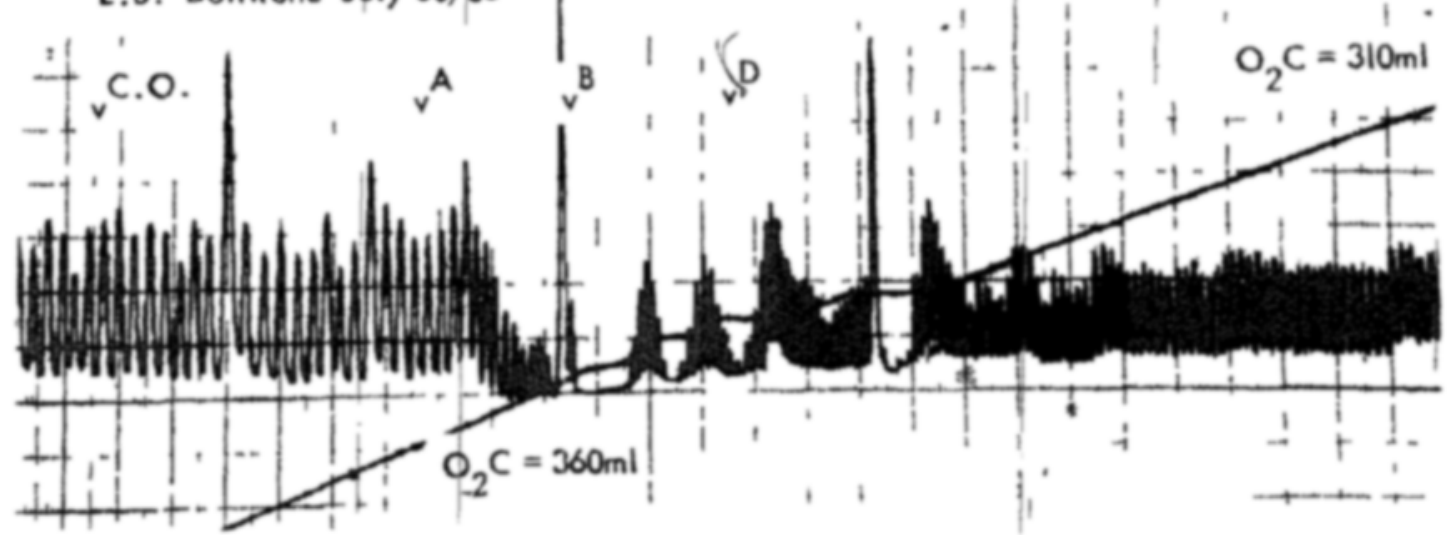

Figure 8 Typical respiration tracings obtained with hexobarbital and Dolitrone. Note the similanty in response with these two agents

imposed small recirculation curve. To correct for the recirculation effect, the curve is extrapolated exponentially from the point $b$ (Fig. 2). Since the same total injected material $(I)$ must come out of the heart as was injected into the inflow tract, this quantity of injected material must be equal to the product of the flow rate $(F)$ multiplied by the concentration of the tagged material $(c)$ in the blood integrated over the entire time of the primary circulation. This is represented by the equation:

$$
I=\int_{0}^{\infty} F c d t
$$

and if $F$, the flow rate or cardiac output, is constant,

$$
I=\int_{0}^{\infty} F c d t
$$

where $\int_{0}^{\infty}$

represents the area under the primary circulation curve and the extrapolation line. This area is equivalent to area $A$ and area $B$ shown in Figure 2. Since the injection dose $I$ is equivalent to the final dilution $C_{f}$ multiplied by the volume $V$, the expression for the cardiac output is: $F=C_{f} V /(A+B)$.

Much has been reported on the accuracy of cardiac output determinations 
by the direct Fick method and by the dye injection method of Hamilton. Any new method must agree closely with these to have experimental validity. In 1949 , Prinzmetal initiated the new approach to the subject by radiocardiography (55). A technique was later developed by Veall and associates (1954) and Huff and associates (1955), for estimating cardiac output by external bodycounting of iodine ${ }^{131}$ following a single injection, using a well-collimated and shielded scintillation detector placed over a fixed site for recording a time concentration curve $(30,31)$. From this, calculation of cardiac output by a modification of the Hamilton dye method has proved to be reliable. Comparison of external body-counting of iodine ${ }^{131}$ with the direct Fick method in men and in dogs by Huff and associates showed no statistically significant difference. MacIntyre has confirmed the accuracy of the radiocardiography method (47), and Huff is extending this work by employing multiple detectors (56).

The probability of random variations in the cardiac output greater than 5 per cent with a standard deviation of 7 per cent in seven cases is less than 5 per cent $(P=<.05)$. This is approximately the same order of significance reported for the Fick method. A consistent difference of 15 per cent is therefore the least change that can be considered to be a significant physiological effect. In this order of significance only consistent changes greater than 20 per cent may be considered as physiological or pharmacological effects or the stroke volume and other parameters derived from the cardiac output. In the tabulated data (Tables X-XVI), the $P$ value for significant changes only are shown. Otherwise, no significance (ns) is recorded

Reviewing mainly the data obtaned on cardiac output with each. drug and each individual, it is evident that major changes did occur, but these were more often not consistently in the same direction. Van Harreveld and Shadle have also noted such changes in haemodynamics by barbiturates and point out that the great complication is produced in the normal curculation by the activity of circulatory reflexes and hormonal mechanisms which affect a number of parameters of the circulatory apparatus simultaneously (57) The analysis of such changes may therefore become very difficult. It is essential, especially in the study of the regulating mechanism of the circulation, to record simultaneously as large a number of dependent variables as possible. These give a more reliable clue to major parameters affected, and the direction of such change. It will be possible only in rare instances to draw conclusions about the mechanisms of circulatory effects from the changes of a single dependent variable like the cardiac output.

\section{Arterial Blood Pressure}

With few exceptions, every drug that was studied in these experiments caused an initial sharp fall in blood pressure which lasted at least a few minutes. This fall was followed by a gradual rise to a lesser depressed level, where it remained until the subject was fully conscious. The initial fall may be considered as primary depression of vasomotor tone, followed rapidly, in most instances, by partial reflex restoration. However, persistent mild depression lasted beyond the time the subject was awake, indicating that some depressant is probably 
present in the circulation in active concentration. This alteration is well known in clinical anaesthesia (58).

\section{Venous Blood Pressure}

The determination of venous pressure has been a neglected aspect of haemodynamic effects of anaesthetic drugs even though its role in surgical shock has been suggested frequently. Moon found that fall in venous pressure, which persists, precedes the fall in arterial blood pressure that is a characteristic of traumatic or haemorrhagic shock-both in expermmental studies and in clinical situations (59). Although venous distension is seen after the administration of vasodılator drugs, it is not accompanied by a decrease in circulating blood volume (27). However, it is seen also with many anaesthetic agents (especially diethyl ether), and particularly with the intravenous anaesthetics, as observed in this study, anc then it is accompanied by a considerable fall in venous pressure. Such a fall is most likely indicative of a reduced circulating blood volume $(60)$. The fall in arterial blood pressure and the rise in pulse rate which accompany or follow this fall in venous pressure can probably be taken as indicative of a significant fall in the circulating blood volume to a level which may produce hypoxia to the heart and other vital organs (brain, liver, kidney).

Although these changes in arterial and venous pressure were well tolerated by the healthy subjects in this study, a critcal disturbance is often evident in the patient who is in poor general health Because venous pressure is usually found to be at its lowest level when a patient is in the supine position, a great fall from this level assumes greater significance and should not be ignored as a sign of advancing shock. The venous pressure changes observed in this study are therefore highly sıgnificant and probably symptomatic of a derangement of haemodynamics which is less obviously indicated by arterial hypotension, by tachycardıa and by an occasional minor electrocardographic alteration.

It was of interest to observe a sudden significant rise in the venous pressure in most experiments immediately upon the subject becoming aware that drug injection "was about to begin. This reaction is clearly evidence of the effect of anxiety on venous pressure The rise may be seen in Figures 5,6 and 7, and is shown on the tables by the range of venous pressures in the control record.

\section{Heart Rate and Electrocardiogram}

There was invariably a marked increase in heart rate above the resting and control levels with all the drugs. This affect was most apparent with Dolitrone, Compound 25398 and hexobarbital. However, no gross disturbances of the PQRST complexes were evident with any of the drugs. Although continuous electrocardiographic recordings were taken throughout this study, it was known that they would be inadequate for estimating the degree of myocardial depression produced by barbiturates, since severe depression of contractile function usually does not lead to any remarkable electrocardiographic chan zes.

In animal studies, Daniels and associates observed that heart fai ure induced by barbiturates alone is primarily the result of their negative inotropic action. However, suppression of autonomic rhythmicity and conduction is responsible 
for cardiac arrest when contractlity is preserved by norepinephrine infusion, and under these curcumstances arrest occurs when excitability is relatively unimparred. They stated that the ability of noradrenaline and isoproterenol to antagonize barbiturate intoxication is probably the result of cardiac stimulation and could not be attributed to peripheral vasoconstriction. They observed further that when these drugs fall to antagonize cardiac depression induced by barbiturates, there is atrio-ventricular block and suppression of sinus pacemaker activity (61).

From the work of Brodie and associates (62) it is evident that the degree of impairment of cardiovascular function is intially determined by the concentration in the blood of the intravenous anaesthetic, and that the metabolic inactivation is not sufficiently rapid to affect the results significantly at the rates of administration employed clinically. This statement unquestionably applies in a similar, but distınguishable degree, to each of the drugs tested in this study.

Since clinical limitations must be respected, we always turn to animal data for guidance and infer the sequence of changes which may occur in man (6372 ). In this respect, it is unfortunate that only a few of the small number of clinical studies $(20,38)$ that have been reported were carefully controlled by lımiting the drugs administered $(73,74,75)$.

The cardiovascular lethal dose of thiopental occurs in dogs with approximately $160 \mathrm{mg}$. per kg. when this agent is infused at the rate of $75 \mathrm{mg}$ per $\mathrm{kg}$ per minute (64). Variation in the extent of redistribution of thiopental into fat may account for some of the variations in the carchovascular lethal doses at different infusion rates This probably applies also to dufferent concentrations of injected drugs. The sequence of events leading to cardiovascular farlure in continuous infusion expermments have been worked out in the dog The following sequence is seen consistently. onset of hypotension soon after infusion is started, progressing rapidly to severe hypotension as the infusion continues. The rate of injection influences the rate of collapse and at critical rates irreversible cardiovascular collapse occurs in spite of artificial respiration. The compensatory tachycardia is not sufficient to compensate for hypoterision The heart rate begins to fall rapidly in the terminal stages $\mathrm{T}$-wave flattening is the earliest significant electrocardiograph change, then further flattening and depression of the ST segment occurs These may not appear until very low blood pressures are reached. Daniels pointed out that barbiturates in quantities not much larger than those producing respiratory arrest, produced marked hypotension in the dog even when hypoxia is prevented (61) Emsloying a "mathematical model of the circulation" Van Harreveld and Shad_e demonstrated that the heart is the primary site of cardıovascular depression due to barbiturates rather than any central or peripheral vasodilating effect (57). If myocardial depression is the principal mechanism whereby cardiovascular function is impaired during barbiturate intoxication, it seems reasonable that much smaller doses of barbiturate may affect cardiac function adversely under appropriate circumstances. This could explain the brief episodes of hypotension often seen with small intravenous doses of barbiturates in intact dogs or in patients The transitory nature of such effects are attributed to compensatory mechanisms, which are present 
until reflex nervous activity is eliminated. However, a study of anaesthetic doses of pentobarbital in dogs attributes hypotensive effects to peripheral vaso-dilatation with no decrease in cardiac output (67). In a study of thiopental anaesthesia in man, a decreased cardiac output with peripheral vasoconstriction and pooling of blood but with no change in arterial pressure was observed. The data from this study confuse the question somewhat surce the patients received premedication with therapeutic doses of opiates, the hiopental was administered in a dilute drip, and electroencephalographic levels rather than actual drug doses were primarily consıdered. Administration by a dilute drip technuque permits compensatory mechanisms to come into play and usually prevents the appearance of acute changes in haemodynamics until very large doses have been administered. The consistent decreases in cardiac output seen by these experimenters therefore is difficult to explain, unless they actually administered very large doses of thiopental or therr patients had impaired cardiac function which was not detected prèoperatively In addition, a potentiatıng depressant effect on the heart by drug combinatons was not ruled out (75).

That anaesthetic levels of thropental actually do depress cardiac function, if compensatory changes cannot occur, has been shown with the heart-lung preparation by Price and Helrich. They demonstrated that changes in slope of the curve relating cardiac output to right arterial pressure caused by thiopental may be used as an index of cardiac depression (76) In the studies of Daniel, despite the infusion of epinephrine in anumals, anaesthetic doses of thiopental stll impaired cardiac function (61)

From work with animals it seems evident that doses of intravenous barbiturates which produced respiratory arrest for more than a brief period directly depress cardiac function. Artficial respiration and stumulation of the central nervous system as by amphetamine alone does not counteract this effect Therefore, cardiac stmulants such as sympathommetic ammes and the catecholamines would seem to be indicated in animals

In applying such information to man it is important to realize that it is essential to limit the use of the short-acting barbiturates to the "sleep dose" with the drug which does not cause more than a few seconds of apnoea. It would be best to employ an' agent which produces hypnosis and some analgesia entrely without apnoea and employ supplementary anaesthetic drugs if greater analgesia and muscle relaxation are necessary for the operative procedure. In this way, cardiovascular function may be preserved in an optmal way without the need for supportive drugs

Since extensive comparative data in humans are lacking for the newer shortactung barbiturates, one must begin with such data as reported in this study and compare them with reports on animal studies and human studies of the individual drugs From these one must attempt to infer those data which are applicable to man Thereafter, clmical data must be collected carefully, with documented notes on relative. potency, duration of sleep for varıous dose levels, rate and concentration of drug administration, rate of onset of sleep and rate of recovery (77). The latter must be related to valud criteria, such as response to name, lucidity, absence of diplopia and dizziness, and ability to concentrate 
mentally. Since repeated doses may be necessary under clinical circumstances, note must be made of "acute" tolerance, cumulation or tachyphylaxis. The degrees of respiratory depression and cardiac acceleration are vital parameters to the clinical anaesthetist and must be diligently recorded. Cardiac arrhythmias, such as ectopic beats and bigeminal rhythm, are easily missed, but these must be carefully sought. Of importance also is the incidence of vagus-like reflexes such as coughing, hiccoughing, sneezing and laryngeal spasm. The protective effect of premedication with belladonna derivatives or a specified technique of anaesthetic induction on these undesirable reactions or reflexes should be sought and clarified. Last but not least, the incidence of inflammatory reactions in the veins must be carefully observed, as must neuromuscular responses to these drugs (twitching, shivering and involuntary spastic movements). Even if an agent fulfils all the desirable anaesthetic criteria, rigidity or twitching in a patient frequently suggests acute hypoxia or toxic reactions in the nervous system and is most undesirable, even if analgesia and hypnosis are optimal.

The data from this study indicate that as far as duration of sleep is concerned for double the sleep dose, the drugs may be divided into two classes: those with over 30 minutes of sleep and more than 20 seconds of apnoea on induction; and those with less than 20 minutes of sleep and less than 10 seconds of apnoea. This division is most evident when the duration of apnoea and sleep are computed on an equal dose basis (Table XIX). In the first group, Compound 25398 is by far the most potent hypnotic, and has the marked disadvantage of causing a prolonged period of apnoea, hypotension and tachycardia. There is essentially no difference between thiopental and thiamylal, although the latter is a somewhat more potent hypnotic, without prolonging the period of sleep, and produces less apnoea on the basis of equal dose administration. The second group of drugs consists of methitural, Dolitrone, hexobarbital and buthalitone. Methi-

TABLE XIX

ANaEsthetic Effect of INTRavenous ANamsthetics

(Calculated on the basis of $500 \mathrm{mg} . / \mathrm{M}^{2}$ of body surface area)

\begin{tabular}{|l|c|c||c|}
\hline \multicolumn{1}{|c|}{ DRUGS } & $\begin{array}{c}\text { Duration of Apnea } \\
\text { seconds }\end{array}$ & $\begin{array}{c}\text { Duration of Sleep } \\
\text { minutes }\end{array}$ & $\begin{array}{c}\text { Sleep Dose } \\
\mathrm{mg} .\end{array}$ \\
\hline LILLY 25398 & 150 & 42 & 273 \\
SURITAL & 29 & 33 & 402 \\
PENTOTHAL & 36 & 34 & 442 \\
NERAVAL & 7 & 19 & 432 \\
DOLITRONE & 8 & 12 & 512 \\
EVIPAL & 7 & 12 & 517 \\
TRANSITHAL & 9 & 5 & 637 \\
\hline
\end{tabular}


tural is the most potent of this group and is very similar to thiopental with respect to the double sleep dose. However, the duration of hypnosis is only approximately 60 per cent of that for thiopental, whereas the period of apnoea is the shortest seen in the whole group with respect to both duration of sleep and the double sleep dose. It would appear from the above findings that methitural has particular advantages over the other three:drugs in that group, if hypnotic potency is desired for short operative procedures, while very short procedures would be best carried out under buthalitone as originally suggested by Weese (69); and Dolitrone has the one advantage of having a definite analgesic effect of its own (13). However, both buthalitone and Dolitrone have a disturbing side effect (twitching or spontaneous movements) which is difficult to tolerate, and Dolitrone has a higher incidence of venous irritation than the other agents (13). Compared to thiopental methitural produces less cardiac acceleration, but the same degree of hypotension, and more yagal-type reactions (coughing, sneezing and hiccoughing) on induction. In animals, a far greater incidence of cardiac arrhythmias (ectopic beats and bigeminal rhythm) is seen with methitural. Arrhythrnias may become evident in clinical anaesthesia with methitural when specifically sought, unless premedication with belladonna derivatives adequately prevent their occurrence. No tolerance or cumulative action for thiopental ( $25 \mathrm{mg} . / \mathrm{kg}$.) and methitural (45 mg. $/ \mathrm{kg}$.) after daily administration for 30 days has been reported $(70)$. There is probably also no tolerance or cumulative action for the other drugs studied.

All the drugs significantly depressed oxygen consumption except hexobarbital, with the greatest decrease produced by thiopental, and the least by Dolitrone. This effect is common to all anaesthetics in varying degree (78). The pulse rate was significantly increased by all the drugs except methitural, with the greatest increase by Dolitrone (39 per cent) and Compound 25398 (36 per cent) and the least by buthalitone ( 10 per cent). All the drugs depressed the blood pressure

TABLE XX

Summary of Significant Mean Percentage Changes in Haemodynamics and Metaibolism duRtNg ACute Study

\begin{tabular}{|c|c|c|c|c|c|c|c|}
\hline & 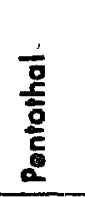 & 葛 & $\frac{\overline{8}}{2}$ & 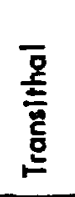 & $\begin{array}{l}\overline{0} \\
\text { : } \\
\text { zo } \\
\end{array}$ & 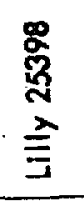 & 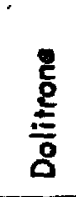 \\
\hline Metabolic Rate & -33 & -29 & -6 & -23 & -25 & -25 & -21 \\
\hline Mean Pulse' Rate & +16 & +14 & +28 & +10 & +3 & +36 & +39 \\
\hline Mean Arterial Pressure & -17 & -7 & -12 & -15 & -17 & -9 & -13 \\
\hline Moan Venous Pressure & -23 & -42 & -17 & -17 & -39 & -43 & -22 \\
\hline
\end{tabular}


to a degree which did not vary greatly among the seven agents. Venous pressure was also reduced by all the drugs by at least 15 per cent, with the east changes observed with hexobarbital and buthalitone (Table XX). None of these changes approached the degree of disturbance which is observed with high spinal anaesthesia $(79,80)$.

\section{Summary and CoNClusions}

The roblems of comparing several anaesthetics are reviewed with particular reference to the effects of premedication, the intrinsic activity of drugs and their affinity for certain tissues and the effect of multiple drug interaction. The physiological and pathological states of those patients who are used as subjects of such studies are essential factors to be clearly determined. The effects of acute tolerance, cumulation and tachyphylaxis were also cited as factors to be considered.

A controlled study of respiratory and haemodynamic parameters was carried out on eight young healthy male subjects in order to determine the principal and statistically significant effects of thiopental, thiamylal, hexobarbital, buthalitone, methitural, Compound 25398 and Dolitrone.

Resting vital signs were initially obtained as well as other vital statistics. Basal metabolism and cardio-respiratory parameters were determined from nomograms related to the restung values These data provided the base line for all control and experimental data Plasma volume and red cell volume were initially determined simultaneously with radioactive iodinated human serum albumin $\left(\mathrm{I}^{131}\right)$ and radioactive chromium ${ }^{51}$, employing the dilution principle. These were checked against venous haematocrit determinations.

Respiration data were obtained on a recording spirometer which also recorded oxygen consumption Cardiovascular data (arterial and venous blood pressure) were recorded via intra-arterial and intravenous needles attached to Statham strain gauges, oscilloscopes and a photographic recorder. The pulse rate and electrocardiogram (lead 2) were also recorded on this systern The cardiac output was estimated by external body-counting of $I^{131}$ following single injections of Rihsa, using a shielded scintillation detector directed over a fixed site on the chest where tissue circulation was minimal, in order to measure primarily the blood flow in the aortic arch. Mean crrculation time and cardiac output were derived from measurements on the recordings, and stroke volume, total peripheral resistance, left ventricular work and intrathoracic blood volume were computed from the above data.

All data were analysed for mean alterations, and statistical significance was computed to determine the probability ( $P$ value) of random variation by the method of the Fisher $t$-test.

The accuracy of the technical procedures employed was reviewed, with particular reference to the newer methods for estımating blood volume, cardiac output and mean curculation time, and was found to compare reliably with that of tried and recognized methods The limitations of clinical studies were also discussed, and data from anımal studies were reviewed with a view to supplementing information on the toxicity and cumulative effects of these drugs. 
A conservative evaluation of the results was essential in this study since it was assumed that deviations of values were normally distributed, and that the study dealt essentially with the healthiest type of subjects one ever encounters in clinical practice The authors also felt, as noted by Johnson and Van Harreveld, that so many factors are involved in cardıo-respiratory regulation. both before and during anaesthesia, that one must be extremely cautous in stating which factor is primarily involved in a change. One must therefore avoid conclusions from individual changes and base critical evaluation on consistent and statistically significant changes in a "homogenous" group, and then perhaps applý these data to an individual

From the experiments, the seven intravenous anaesthetics studied could be divided into two main groups: those that produced a prolonged depression of respiration and circulation; and those that produced a brief depression of these parameters. Compound 25398, thiamylal and thiopental belong to the first group, while methitural, Dolitrone, hexobarbital and buthalitone belong to the second. All the drugs except hexobarbital depressed oxygen consumption. Tachycardia was a significant finding with all the drugs except methitural and acute hypotension (arterial and verous) was a consistent finding with all the drugs. Considering all the parameters, myocardial depression was induced by the first group, but this was not consistently manifested by a decrease in the cardiac output.

From this investigation, it is difficult to choose among the agents and state which disturbs human physiology to the least extent while providing clinically adequate hypnosis. It is - only possible to state the advantare of a particular agent with reference to a specific patient, a specific type o: operation and a specific clinical situation On this basis, extensive clinical experience with one agent is probably the most reliable method of managing most situations, for then the problem comes down to a matter of dosage, concentration of the drug, and rate of its administration.

\section{ACKNOWLEDGMENTS}

The authors wish to thank T E Merrman, M.D, F.R CP.(C), Mr. and Mrs $\mathrm{R}$ L. J Rainbow and Mr. K Varley of the Cardio-Pulmonary Laboratory for assistance with recordings of vital signs, T. A. Watson, M.D, Esther M. Brown, M D., Sylvia Fedoruk, M A and William B Reid, Ph D, of the Saskatchewan Cancer Service for advice and technical assistance with estumations of blood volume and cardiac output, and the following medical students who volunteered and co-operated throughout these studies Alfred Bludoff, Leonard Cox, Wilfred Hathway, Norwood Kavanagh, Donald Schmidt, Ernest'Baergen, Peter Block and Archibald Levy.

\section{RÉSUMÉ}

Nous avons étudié les problèmes que pose la comparason de plusieurs anesthésiques en prêtant une attention particuhère aux effets de la prémédıcation, à l'activité intrinsèques des médıcaments et à leur affinité pour certains tussus, puss, enfin, à l'effet de l'interaction de plusieurs médicaments Il nous faut donner des précisions su la condition physio-pathologique des malades chez qui on a 
employé ces médicaments pour les étudier. Nous avons également mentionné comme facteurs non négligeables. la tolérance aigue, l'accumulation et la tachyphylaxie.

Nous avons fart, chez huit jeunes sujets mâles en sarité, une étude, avec contrôles, des paramètres respiratorres et hémodynamiques pour tabuler les effets principaux et les effets présentant un intérêt statistique sur le thiopental, le thiamylal, l'hexobarbital, le buthalıtone, le methitural, le composé 25398 et le dolitrone.

Nous avons d'abord cherché les signes vitaux à l'état de repos de même que d'autres statıstiques vitales Le métabolısme de base et les paramètres cardırespiratoires ont été trés des nomogrammes donnant les valeurs à l'état de repos Ces résultats nous ont fourm les bases pour les données de contrôle et expérımentales Les volumes plasmatique et globulaire ont été recherchés simultanément au début avec le sérum albumine humain odé radıoactif $\left(\mathrm{I}^{131}\right)$ et le chromium radioactifí en employant le principe de dılution. Ces deux résultats ont été comparés aux déterminations de l'hématocrite sur le sang veıneux

Au sujet de la respiration, les données ont été obtenues au moyen d'un spiromètre enregistreur renseignant également sur la consommation d'oxygène. Les renseignements sur le système cardiovascularre (pression artérielle et veineuse) ont été obtenus et enregistrés au moyen d'aiguilles placées dans les artères et dans les veines et relıées au manomètres Statham, aux oscilloscopes et à un enregistreur photographique

Le rythme cardıaque et l'électrocardiogramme (en deuxıème dérivation) ont également été enregistrés sur le même système Le débit cardraque a été évalué de l'extérieur de l'organisme en repérant l' $I^{131}$, après une seule injection de Rihsa, et en promenant un détecteur à champ scintillant au-dessus d'un endroit précis sur le thorax où la circulation tissulaire est réduite de façon à obtenir principalement le débit sangum de la crosse aortique La moyenne du temps de circulation et le débit cardiaque ont été obtenus de ces enregistrements, et le débit systolique, la résistance périphérique totale, le travall du ventricule gauche et le volume de sang intrathoracıque ont été calculés d'après ces données

Tous les résultats ont été analysés pour trouver la moyenne des modifications et la significatıon statıstıque a été calculée pour obtenir la probabilité (la valeur $P$ ) de variation isolée par la méthode de Fisher

Nous avons contrôlé la précisıon des techniques employées, plus particulièrement les techniques nouvelles pour mesurer le volume sanguin, le débit cardiaque et la moyenne du temps de circulation et nous pouvons affirmer que les résultats sont comparables à ceux obtenus par d'autres techniques éprouvées et reconnues Nous avons également discuté des limites des études clinıques et les résultats des études fartes sur l'anumal ont été étudiés dans le but d'obtenir des rensergnements additionnels sur la toxicité et les effets cumulatifs de ces médıcaments

Dans cette étude, il était essentiel d'avoir une évaluation conservatrice des résultats car nous présumıns que la déviation des résultats étart répartie normalement et que l'étude a été farte sur des sujets les plus en santé qu'on puisse trouver en clinique. Les auteurs ont également eu la convictıon, comme l'affirment Johnson et Van Harreveld, qu'il y a tellement de facteurs impliqués dans 
le maintren de l'équilibre cardiorespiratoire, aussi bien avant que pendant l'anesthésie, qu'il faut être très prudent avant d'affirmer que tel ou tel facteur est primitıvement responsable d'un changement Conséquemment, il faut éviter de conclure d'après des changements individuels et baser une évaluation critique s s.r des changements considér ables et d'importance statistique observés sur un grou'e homogène et après, peut-être, comparer ces données à l'individu.

D'après ces expériences, on peut diviser en deux groupes les sept agents anesthésiques étudiés, il s'agit d'agents administrés par voie endoveineuse: ceux qui ont entraîné une dépression prolongée de la respiration et de la circulation et ceux qui ont entraîné une brève dépression sur ces paramètres. Le composé 25398 , le thiamylal et thopental appartiennent au premier groupe, tandis que le methitural, le dolitrone, l'hexobarbital et le buthalitone appartiennent au dernier groupe. Tous ces médicarnents diminuent la consommation d'oxygène à l'exception de l'hexobarbital. On a observé une tachycardie importante à la suite de l'administrảton de tous ces médicaments à l'exception du méthitural et une hypotension marquée (artérielle et veineuse) à la su.te de l'administration de chacun de ces médicaments En analysant tous les résultats, on note que les médicaments du premier groupe ont causé une dépression myocardique mais elle n'a pas toujours été accompagnée d'une dıminution du débit cardiaque.

Après cette enquête, il demeure difficile d'affirmer lequel de ces agents cause le moins de changement dans la physiologie humanie tout en procurant une hypnose adéquate cliniquement. Il nous est possible tout au plus de mentionner la supériorité d'un agent en particulier pour tel ou tel malade, ou encore pour telle sorte d'opération ou'pour une situation clinique particulière. En se basant sur ces faits, nous pouvons affirmer qu'une longue expérience clinique avec un agent demeure probablement le facteur le plus sûr pour contrôler toutes les éventuálités, car le problème se résume à une question de dosage, de dilution de médicament et de vitesse d'administration.

\section{REFERENCES}

1 KeAtişG, V Anaesthesıa Accidents, pp 136 et seq London: Lloyd-Luke (1956).

2 Stephenson, H E, Jr, Reid, L C \& Hinton, J W Some Common Denominators in 1200 Cases of Cardiaq Arrest Ann Surg, 137731 (1953)

3 BEecher, H K \& ToDd, D P A Study of the Deaths Associated with Anaesthesia and Surgery Ann Surg., 140 2(1954)

4 Edwards, G Morton, H J V, Pask, E A \& Wylie, W D Deaths Associated with Anaesthesia Anaesthesia, 11 194 (1956)

5 Editorial Influence of Opiates upon Experimental Results Anesthesiology, 17616 (1956)

6 Wangeman, C P \& Hawk, M H The Effects of Morphine, Atropine and Scopolamine on Human Subjects Anesthesiology, 324 (1942)

7 Cohen, E N \& BeEcher, H K Narcotics in Preanaesthetic Medication-Controlled Study Report to Council on Pharmacy and Chemistry J A M A, 1471664 (1951)

8 Dobkin, A: B, Gilbert, R G B \& Melville, K I Chlorpromazine Review and Investigation as a Premedicant in Anesthesia Anesthesiology, $17 \quad 135$ (1956)

9 Helrich, M, Eckenhoff, J E, Jones, $R$ E \& Rolph, W D, Jr Influence of Opiates on the Respiratory Response of Man to Thiopental Anesthesiology, 17459 (1956)

10 Dobkin, A B, Wyant, G M \& Dyck, F Clinical Evaluation of Methyprylon (Noludar) as a Preanaesthetic Sedatıve Hypnotıc Canad Anaesth Soc J, 427 (1957). 
11. Swernlow, M \& Newman, J. Some Effects of Premedication Brit J Anaesth, 2966 (1957).

12. Dundee, J. W., Price, H L \& Drrpps, R. D. Acute Tolerance to Thiopentone in Man Bnt. J Anaesth, 8344 (1956)

13 Wyant, G M, Kildouff, C J, Merriman, J E \& Dobkin, A B Evaluation of Dolitrone Canad Anaesth Soc J, 3291 (1956)

14 Wyant, G M, Dobkin, A B \& Kilduff, C J The Effect of Buthalitone Sodium (Transithal@) on Liver and Kidney Function in Man.. Canad Anaesth Soc J, 421 (1957).

15 Radford, E P, Jr Ventilation Standards for Use in Artıficial Respiration J Appl Physiol, 7451 (1955)

16 JACKson, C E Nomogram for Simple Calculation of Cardiac Output Circulation, 11. 635 (1955)

17. Stead, E. A, Warren, J V, Merrill, A J \& Braninon, E S The Cardiac Output in Male Subjects as Measured by the Technique of Right Atrial Catheterization Normal Values with Observations on the Effect of Anxiety and Tiling J Clin Investigation, 24326 (1945)

18 Hickam, J B, Cargill, W H. \& Golden, A Cardiovascular Reactions to Emotional Stımuli. Effect on Cardiac Output, Arteriovenous Oxygen Difference, Arterial Pressure and Peripheral Resistance J Chn Investigation, 27290 (1948)

19 Henschel, A. B., Wyant, G M, Dobkin, A B \& Henschel, E O. Posture as It Concerns the Anesthesiologist. A Prelmminary Study Anesth and Analg., 3669 (1957)

20 O'Mullane, E J The Investigation of a Short-Acting Barbiturate, Illustrating the Fallacies of Climical Impressions Brit J Anaesth, 2971 (1957)

21. Gray, S J \& Sterlung, K. Determination of Curculating Red Cell Volume in Man by Radroactive Chromum J Clin Investigation, 29 1604 (1950)

22. Sterling, K. \& Gray, S J Determination of Circulating Red Cell Volume in Man with Radioactive Chromium J Clin Investigation, 29 1614 (1950)

23. Crusperl, $K$ R, Ponter, B \& Nieset, $R$ T Studies of Plasma Volume Using Human Serum Albumin Tagrend with Radioactıve Iodıne ( I $\left.^{131}\right)$ J Clin Investigation, 29. 513 (1950).

24 Storaasli, J P, Krieger, H, Friedeli, H L \& Holden, W D Use of Radioactive Iodinated Plasma Protein in Study of Blood Volume Surg, Gynec \& Obst, 91458 (1950).

25 Erickson, J R, McCormick, J B \& Seed, L Improved Method for Determination of Blood Volume Using Radıoactive Iodınated Human Serum Albumın Science, 118595 (1953)

26 Gray, S J \& Frank, H The Simultaneous Determination of Red Cell Mass and Plasma Volume in Man with Radioactive Sodium Chromate and Chromic Chloride J Clin Investigation, 321000 (1953)

27 Dobxin, A B Effect of Chlorpromazine on Blood Volume, Venous Pressure and: Circulation Time Estımations Anaesthesia, in press (1957)

28 Lyons, R H, Kennedy, J A \& Burweli, C S The Measurement of Venous Pressure by the Direct Method Am Heart J, 16 675 (1938)

29 Fry, D L, Noble, F W \& Mallos, A S An Evaluation of Modern Pressure Recording Systems Clin Res, 5 40 (1957)

30 Veall, N, Pearson, J D, Hanley, T \& Lowe, A E A Method for the Determination of Cardiac Output (Prelıminary Report), pp 183-192 Proc 2nd Radioısotope Conference, Oxford, July 19-23 (1954) London Butterworth's Scientific Publications (1954)

31 Huff, R L, Feller, D D, Judd, O J \& Bogardus, G. M. Cardiac Output of Men and Dogs Measured by in vivo Analysis of Iodinated (I131) Human Serum Albumin Circ Res, 3564 (1955) 
32 Shipley, R A, Clark, R E, Liebowitz, D \& Krohmer, J S Analysis of the Radiocardiogram in Heart Fallure. Curc Res, 1 428 (1953).

33 Shipley, R A \& Cilark, R E Measurement of Curculation Times with NaI131. Circ. Res, 4456 (1956)

34 Crvin, $\mathrm{K}$ Calculation of Cardiac Output by Estimation with Dilution Method. Acta Physiol Scandinav, 1957 (1949)

35 Hammton, W F, Moore, J W, Kinsman, J M \& Sparling, R G Studies on the Crrculation IV Further Analysis of the Injection Method and of Changes in Hemodynamics under Physıological and Pathological Conditons Am J Physiol, 99534 (1932)

36 ShIPLEY, R A Personal communication

37 Apiera, A Total Peripheral Resistance Formula Skandinav. Arch f Physiol, Suppl. 16 1 (1940), quoted by R Shackman et al

38 Johnson, S. R. The Effect of Some Anaesthestic Agents on the Circulation in Man. Acta chir Scandinav, Suppl 158 (1951

39 Remington, J W \& Haminton, W F The Evaluation of the Work of the Heart. Am J Physiol, 150267 (1947)

40 Stewart, G N The Pulmonary Circulation Time, the Quantity of Blood in the Lungs and the Output of the Heart Am J Physiol, 5820 (1921)

41 Newman, E. V et al The Dye Dilution Method for Describing the Central Circulation: An Analysis of Factors Shaping the Time Concentration Curves Circulation, 4735 (1951)

42 Kattus, A. A, Rivin, A U, Comen, A \& Sofia, G S Cardiac Output and Central Volume as Determined by Dye Dilution Curves Restıng Values in Normal Subjects and Patients with Cardiovascular Disease Circulation, 11 447.(1955)

43 Hamilon, W F The Fhysiology of the Cardiac Output Circulation, 8527 (1953).

44 Ebert, R V, Borden, C W, Wells, H S \& Wrison, R H Studies of the Pulmonary Circulation I The Circulation Time from Pulmonary Artery to the Femoral Artery and the Quantity of Blood in the Lungs in Normal Individuals J. Clin Investigation, 28 1134 (1949)

45 Macintyre, W J \& Leonards, J R A Method for Contınuously Recording the Disappearance of Radioactive Tracers from Curculating Blood. Curc. Res, 3. 14 (1955).

46 Pritchind, W H, Moir, T VV \& Macintyhe, W J Measurement of the Early Disappearance of Iodinated ( ${ }^{131}$ ) Serum Albumin from Circulating Blood by a Continuous Recording Method Circ Res , 319 (1955)

47 Macintyre, W J, Pritchard, W H, Moir, T W \& Friedeld, H Some Studies on the Determination of Cardiac Output by the Dilution Method without Arterial Sampling Abstract Internat J Appl Radiation \& Isotopes, 1 136 (1956).

48 Hicks, D A, Hope, A, Turnbull, A L \& Verel, D The Prediction and Estimation of Normal Blood Volume Chn Sc, 15557 (1956)

49 Hamilton, W F et al Comparison of the Fick and the Dye Injection Method of Measuring the Cardiac Output in Man Am J Physiol, 153309 (1948)

50 ConN, H、 L Accuracy of a Radiopotassium Dilution (Stewart Principle) Method for Measurement of Cardiac Output J Appl Physıol , 7542 (1955)

51 Karpeles, $L$ M \& Huff, $R$ L Blood Volume of Representative Portions of the Musculo-Skeletal System in Man Circ Res, 3483 (1955)

52 Macintyre, W J, Prutchard, W H, Eckstein, R W \& Friedeld, H. L The Determination of Cardiac Output by a Contınuous Recording System Utilizing Iodinated (I ${ }^{131}$ ) Human Serum Albumin I Anmal Studies Circulation, 4552 (1951)

53 Pritchard, W H, Macintyre, W J, Schmidt, W C, Bronfman, B L \& Moore, D J The Determination of 'Cardiac Output by a Contmuous Recording System Utılizing Iodinated (I131) Human Serum Albumin II Clinical Studies Circulation, $6 \quad 572$ (1952)

54 Lammerant, J, Sprumont, P \& De Visscher, M Registration of Intracardiac Blood 
Flow for Man A Radioisotope Dilution Technique Arch internat de physiol et de brochem, 74 I (1956).

55 Prinzmetal, M, Corday, E, Sprutzler, R J \& Flieg, W Radiocardiography and Its Clinical Application J A M A, 139. 617 (1949).

56 Huff, $R$ L, Parfish, D \& Crocketr, W Simultaneous Recordıngs of Radiation (I131) Transients from Four Crystal Detectors on the Anterior Thoracic Wall. Bulletin of U.S A E C, No AT( 45-1)-978

57 Van Harreveld, A \& Shadle, O W On Hemodynamics Arch internat de physiol, 59165 (1951)

58 Elder, J D, Jr, Nagano, S M, Eastwood, D W \& Harnagel, D Circulatory Changes Associated with Thiopental Anaesthesia in Man Anesthesiology, 16394 (1955)

59. Moon, V H Shock Its Dynamics, Occurrence and Management, pp 24 et seq Philadelphia Lea and Febiger (1942)

60 Pierce, V K, Boyan, C P \& Mastersjn, J G Studies on Venous Blood Pressure in Patients Undergoing Major Surgical Procedures Surg Gynec \& Obst, 96310 (1953)

61 Daniel, E E, Fulton, J B, Hiddleston, M, Martin, $W$ \& Foulks, J G An Analysıs of the Mechanism of Barbiturate Induced Cardiovascular Depression and Its Antagonism by Sympathommetic Ammes Arch internat de pharmacodyn et de therap, 108457 (1956)

62 Brodie, B. B, Bunns, J J, Mark, L C, Lief, P A, Bernstein, E \& Papper, E M The Fate of Pentobarbital in Man and Dog and a Method for Its Estimation in Biological Material J Pharmacol \& Exper Therap, 10926 (1953).

63 Wyngaarden, J B, Woods, L A, Ridley, R \& Seevers, M H. Anesthetic Properties of Sodium 5-Allyl 5-(1-Methyl-butyl)-2 Thiobarbiturate (Surital) and Certain Other Thobarbiturates in Dogs J Pharmacol \& Exper Therap, 95322 (1949)

64 Woods, L A, Wynganrden, J B, Rennick, B \& Seevers, M H Cardiovascular Toxicity of Thiobarbiturates Comparison of Thiopental and Surital in Dogs J Pharmacol \& Exper Therap, 95328 (1949)

65 Prime, F. J \& Gray, T C The Effect of Certain Anaesthetic and Relaxant Agents on Circulatory Dynamics Bnt J Anaesth, 24 101 (1952)

66 Boniface, K G \& Brown, J M Quantitative Evaluation of Cardiovascular Stimulant Drugs in Barbiturate Depression of the Heart of the Dog Anesthesiology, 1423 (1953)

67 Imig, C J, Randali, B F \& Hines, H M Effect of Pentobarbital Sodium Anesthesia upon Volume Blood Flow, Arterial Pressure and Heart Rate Proc Soc Exper Biol \& Med, 829 (1953)

68 Greisheimer, E M, Ellis, D W, Baien, H N, Ring, G C, Makarenko, L \& Graziano, J Cardiac Output by Cuvette Oxımeter under Thiopental Am J Physiol, 175171 (1953)

69 WEEsE, M \& Kcss, F H Ueber en neues Ultrakurznarkoticum Deutsche med Wochnschr, 79601 (1954)

70 Irwin, S, Stagg, R D, Dunbar, E \& Govier, W M Methitural, a New Intravenous Anaesthetic Companson with Thiopental in the Cat, Dog and Monkey J Pharmacol \& Exper Therap, 116317 (1956)

71 Blake, M W \& Perlman, P L Metabohism of the Ultrashort-Acting Thiobarbiturate Methitural (Neraval) J Pharmacol \& Exper Therap, 117287 (1956)

72 Greisheimer, E M et al Cardiovascular Responses to Several Ultra Short Acting Barbiturates Anesthesiology, 17385 (1956)

73 Shackmav, R, Graber, I G \& Melrose, D G Haemodynamics of Surgical Patient under General Anaesthesia Brit J Surg, 40193 (1952)

74 Lee, G de J, Churchill-Davidson, H, Miles, $B$ E \& dF Wardener, $H$ E Circulatory Effects of Prolonged Light Anaesthesia in Man Clin Sc, 12169 (1953)

75 Etsten, B \& LI, T H Hemodynamic Changes durng Thopental Anesthesia in Humans Cardiac Output, Stroke Volume, Total Peripheral Resistance and Intrathoracic Blood Volume J Clin Investigation, 34500 (1955). 
76. Price, H. L \& Hel.ruch, M. The Effect of Cyclopropane, Diethyl Ether, Nitrous Oxide, Thiopental and Hydrogen Ion Concentration on the Myocardial Function of the Dog Heart-Lung Preparation. J. Pharmacol. \& Exper. Therap, 115. 206 (1955).

77 O'Hertihy, D B., Nishimura, N., Little, D. M., Jr., \& Tovell. R. M. The Clinical Usage of Neraval. Canad. Anaesth. Soc. J., 3. 326 (1956).

78 Shackman, R., Graber, G. I. \& Redwood, C. Oxygen Consumption and Anaesthesia. Clin. Sc, 10. 219 (1951).

79. Lynn, R. B., Sancetta, S M, Simeone, F A. \& Scotr, R. W. Observations on the Circulation in High Spmal Anesthesia Surgery, 32195 (1952)

80. Sancetta, S M, Lynn, R. B Simeone, F A \& ScotT, $R$ W Studies on Hemodynamic Changes in Humans following Induction of Low and High Spinal Anesthesia. Circulation, 6: 559 (1952) 